\title{
Estado, mercado y usos indígenas de la tierra: La Barrancosa (Buenos Aires, 1863-1906)
}

\author{
por \\ Luciano Literas ${ }^{1}$ \\ CONICET - Universidad de Buenos Aires, \\ Facultad de Filosofía y Letras, Instituto de Ciencias Antropológicas
}

\begin{abstract}
El siguiente artículo analiza la posesión y el uso indígena de la tierra en la provincia de Buenos Aires (Argentina), en el marco de la organización estatal y la formación del mercado de tierras en la segunda mitad del siglo XIX. Para hacerlo trata el caso de la tribu de Melinao y las tierras que poblaban en La Barrancosa (Bragado, Buenos Aires), cuya propiedad recibieron como donación en 1863. El análisis se basa en la triangulación de un heterogéneo conjunto de fuentes documentales sobre población, servicio de armas, parentesco, tierras, producción y comercio.
\end{abstract}

Palabras Clave: Pampas y Norpatagonia; pueblos indigenas; «indios amigos»; mercado de tierras; organización estatal.

Cómo CITAR este artículo / Citation: Literas, Luciano, "Estado, mercado y usos indígenas de la tierra: La Barrancosa (Buenos Aires, 1863-1906)", Revista de Indias, LXXX/280 (Madrid, 2020): 781-814. https://doi.org/10.3989/revindias.2020.022.

\section{INTRODUCCIÓN}

Durante la segunda mitad del siglo XIX tuvieron lugar en la provincia de Buenos Aires (Argentina) un conjunto de experiencias asociadas al acceso a la propiedad y el uso de la tierra cuyo protagonista fue la población indígena de las llanuras pampeanas. Esto sucedió en el marco del avance fronterizo del Estado argentino hacia el sur y la construcción de un mercado de tierras - que tuvo como acontecimiento más significativo a las campañas de ocupación

\footnotetext{
1 lucianoliteras@gmail.com, ORCID iD: https://orcid.org/0000-0001-7156-9497
} 
militar denominadas Conquista del Desierto (1879-1885) - y fueron resultado de las heterogéneas trayectorias de inserción indígena en la frontera.

Muchas de estas experiencias tuvieron inicio en las donaciones de propiedad que entre 1863 y 1869 hizo el gobierno de la provincia de Buenos Aires a varias poblaciones indígenas con respecto a las tierras que habitaban. Esto se hizo bajo el rótulo jurídico de «al cacique y su tribu», sin más precisiones sobre derechos, obligaciones ni distribución - solo se especificó que las tierras no podían enajenarse por diez años-, e incluyó a las «tribus» ${ }^{2}$ del cacique Melinao en La Barrancosa (Bragado), Ancalao y Guayquil en el arroyo Pareja y Nueva Roma (Bahía Blanca), Coliqueo en Tapera de Díaz (Los Toldos), Rondeau en Cruz de Guerra (Veinticinco de Mayo) y Raninqueo y Tripailaf en La Verde (Nueve de Julio) (ver mapa 1). Hace algunos años, en el marco de la biografía de los líderes indígenas de las Pampas y Norpatagonia, Meinrado Hux ${ }^{3}$ advirtió las conexiones de estas donaciones con la diplomacia fronteriza y las prestaciones militares en órbita estatal - dos rasgos característicos de los grupos denominados estatalmente como «indios amigos»—; algo confirmado por estudios posteriores ${ }^{4}$. Sin embargo, su análisis reeditó uno de los problemas centrales de las investigaciones previas sobre la población criolla de la campaña bonaerense en el siglo XIX: que la propiedad de la tierra no explica la posesión ni menos aún su uso ${ }^{5}$.

El propósito del siguiente artículo es avanzar en la comprensión de la posesión y el uso indígena de la tierra en la provincia de Buenos Aires, en el marco de la organización estatal y la formación del mercado de tierras. Para hacerlo toma el caso de la tribu de Melinao y las tierras que poblaban en el paraje La Barrancosa, en el cuartel séptimo del partido de $\mathrm{Bragado}^{6}$, cuya

2 Al menos desde los estudios de Martha Bechis en la década de 1980, la antropología y la historia argentina discuten el significado del término «tribu» para las Pampas y Norpatagonia. Esa fue la denominación empleada en el siglo XIX para los grupos de diferentes parcialidades que se insertaron en las tramas de relaciones sociales, políticas y económicas del Negocio Pacífico de Indios y que posteriormente, en algunos casos, recibieron donaciones de tierras. Es decir, «tribu» no es sinónimo de «nación» ni «grupo étnico» y alude más bien, a una entidad política fraguada al calor de las relaciones interétnicas de frontera, conformada por segmentos de la sociedad indígena pampeano-patagónica. Aquí se utilizará el término «tribu» con ese sentido.

3 Hux, 2004 [1992]; 2007.

4 Por ejemplo los estudios de los casos de Coliqueo (Fischman y Hernández, 1990), Maycá (Lanteri et. al., 2011. Barbuto, 2014. Lanteri y Pedrotta, 2018), Rondeau (Literas, 2015), Melinao (Literas, 2016), Ancalao (Martinelli, 2017; 2018) y Raninqueo (Literas, 2018a) y abordajes en perspectiva comparativa (de Jong, 2015. Literas y Barbuto, 2015).

5 Esto se observa en los numerosos trabajos de Juan Carlos Garavaglia, Jorge Gelman, María Elena Infesta, Marta Valencia, Guillermo Banzato y María Fernanda Barcos, entre otros.

6 Partido es la jurisdicción política que divide el espacio provincial. Los cuarteles, a su vez, subdividen a los partidos en su ámbito estrictamente rural, más allá de los ejidos urbanos. 
propiedad recibieron en carácter de donación en 18637. El análisis dirige la mirada más allá de la propiedad — aspecto sin duda más visible y diáfano en las fuentes documentales - y hace foco en la posesión y el uso de la tierra. Esto implica trascender el conjunto de vínculos, recursos y capitales involucrados en la negociación y obtención de la propiedad de la tierra - aspectos sobre los que versó la mayor parte de la escasa historiografía sobre el problema de estudio - e intentar reconstruir e indagar cómo se habitó, distribuyó y explotó. Aquí una primera hipótesis es que el parentesco explica en gran parte las modalidades de posesión y uso, así como los procesos de fragmentación y enajenación no indígena observables en décadas posteriores. La propiedad no explica la posesión, pero al menos en algunos casos sí sucede a la inversa ${ }^{8}$.

La estrategia metodológica se apoya en la triangulación constante de documentos de diferente origen, factura, propósito y características, a fines de identificar individualmente a la población adjudicataria de la donación - encapsulada en el término «tribu»-, reconstruir sus itinerarios, vínculos interpersonales, redes de relaciones más amplias y vínculos de diferente tipo con la tierra donada a la tribu en 1863, a lo largo de las cuatro décadas posteriores.

Un primer conjunto de fuentes son militares y consisten en las listas de revista y de racionamiento ${ }^{9}$ de la población indígena militarizada en el marco de la unidad socio-política llamada «tribu de Melinao», desde 1852 hasta su licenciamiento en 1869, además de la movilización coyuntural de 1874 en función de la incursión del comandante Hilario Lagos a territorio ranquel. Ya se ha hablado de las características de este tipo de fuentes, así como de sus alcances, límites y recaudos para el análisis ${ }^{10}$. Empero es preciso recordar la principal virtud de este registro: permite identificar y localizar a los hombres militarizados en los cuerpos indígenas y sus familias - la «chusma» para la historiografía clásica - con una periodicidad regular y en un marco temporal

7 Ya se han reconstruido los orígenes de esta donación y un primer esbozo de distribución haciendo énfasis en el papel de los caciques y la reformulación que implicó en el liderazgo político (Literas, 2016).

8 El estudio pionero de Fischman y Hernández (1990) sobre la tribu de Coliqueo advirtió que algunas de estas experiencias de donación no prosperaron, en razón de la ocupación criolla, la parcelación y fragmentación de la posesión, la introducción de formas jurídicas no indígenas y la ulterior enajenación de la tierra.

9 Desde hace unos años se ha identificado y analizado este tipo de documentos en diferentes archivos nacionales, provinciales y municipales de Argentina con el propósito de reunir, sistematizar y analizar el corpus generado para la militarización, el racionamiento y/o el presidio de la población indígena de las Pampas y Norpatagonia en la segunda mitad del siglo XIX (Literas y Barbuto, 2017).

10 Literas, 2016. Literas y Barbuto, 2017. 
amplio. Esto tiene especial relevancia heurística para una perspectiva de análisis que hace énfasis en las articulaciones entre liderazgos políticos y bases sociales, poniendo el foco en las mayorías historiográficamente invisibilizadas o destinadas al epígrafe «del número y el anonimato» ${ }^{11}$.

Un segundo conjunto de fuentes son las demográficas, específicamente las planillas de los censos nacionales de 1869 y 1895 , los registros vecinales de los cuarteles de Bragado de 1890 y 1891, y los registros cívicos de 1885, 1891 y del 1897 a $1900^{12}$. Su triangulación con las listas de revista y de racionamiento es clave a la hora de generar información demográfica de los individuos, así como precisar las relaciones de parentesco, y la conformación y localización de las unidades domésticas. Al respecto los registros vecinales son especialmente útiles porque son una relación de las unidades domésticas asentadas en las tierras de la tribu en forma de chacras y puestos con la correspondiente descripción de sus integrantes y ocupaciones laborales ofreciendo también, en consecuencia, indicios sobre el uso de la tierra.

Un tercer conjunto de fuentes son las asociadas a la explotación económica de la tierra y la comercialización de sus productos: las marcas de señales de ganado y guías de campaña tramitadas en el ámbito municipal a lo largo de la segunda mitad del siglo XIX. Ellas fueron los principales instrumentos escritos para regular la cría y la comercialización del ganado, aún en uso en la actualidad ${ }^{13}$. La primera era el símbolo que identificaba al propietario del ganado - las más de las veces con características gráficas alusivas a las letras iniciales de su nombre o a la especie de animal marcado- y la segunda era el boleto emitido en función de su compra-venta y circulación.

Un cuarto y último conjunto de fuentes es de tipo catastral: las mensuras de La Barrancosa elaboradas en 1903 y 1906, el plano generado por una inspección municipal de 1912 y el registro catastral del partido de 1920. La relevancia de dichos documentos, en este caso, radica en esbozar de modo indiciario cómo la población liderada por el cacique Melinao fraccionó y distribuyó la posesión y el uso de la tierra al incluir, por ejemplo, el relevamiento de los asentamientos.

Todas estas fuentes ofrecen posibilidades en virtud de identificar y reconstruir actores, relaciones y prácticas sociales, políticas y económicas. Como se adelantó, su triangulación es insoslayable porque cada una de ellas, dado su

11 Ginzburg, 2011 [1976]: 21.

12 Los registros vecinales y cívicos pertenecen al AHMB a los cuales se accedió y consultó gracias a la valiosa orientación y ayuda de su directora, Marcela Coñequir.

13 Ya se ha analizado este tipo de fuentes para el caso de las tierras de la tribu de Rondeau (Literas, 2015). 
MAPA 1. LAS INICIATIVAS DE DONACIÓN DE TIERRAS A «INDIOS AMIGOS» EN LAS FRONTERAS DE LAS PAMPAS Y NoRPATAGONIA, SEGUNDA MITAD DEL SIGLO XIX

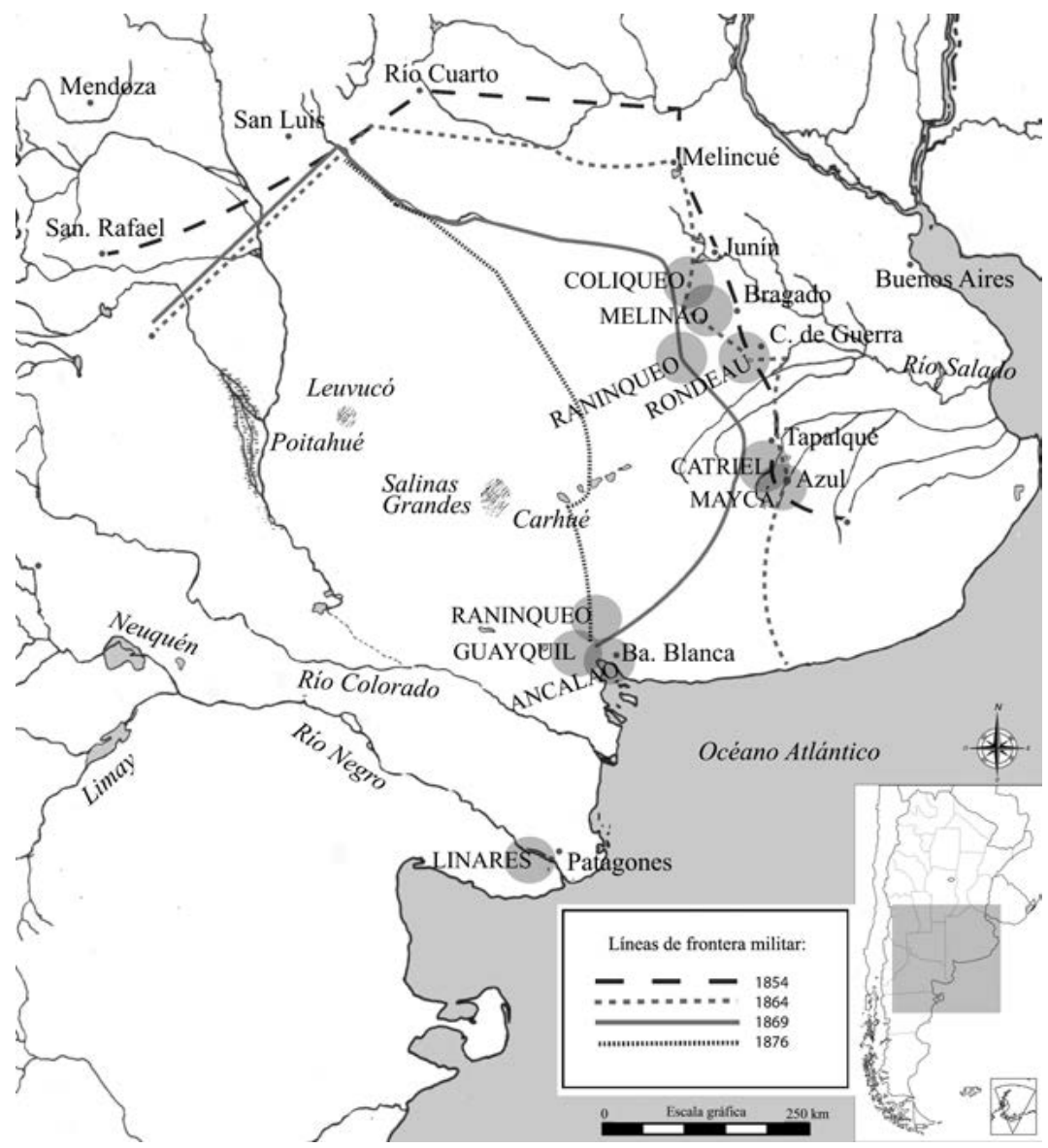

Fuente: Literas y Barbuto, 2015: 164.

origen, finalidad y características, dan cuenta solo de una porción de la reali$\mathrm{dad}^{14}$. A su vez, las fuentes tienen claras limitaciones no solo por haber sido producidas por los funcionarios del Estado - aunque esto no obture abordar la agencia indígena - y porque la organización institucional sobre la que se elaboraban era en muchos aspectos precaria, sino porque como veremos, numero-

14 Ginzburg y Poni, 2004 [1979]. 
sas prácticas se dieron por fuera de las regulaciones, observancias y protocolos burocráticos del Estado.

\section{MilitARIZACIÓN, PARENTESCO Y ACCESO A LA TIERRA}

Como se adelantó, las donaciones de tierras en el ámbito rural a los «indios amigos» se dieron entre 1863 y $1869^{15}$. Por entonces estos grupos tenían una significativa relevancia en la administración militar fronteriza. La batalla de Pavón (1861), la Guerra del Paraguay (1864-1870) y las «montoneras» en Cuyo agudizaron las exigencias militares del Estado y redirigieron coyunturalmente a las fuerzas milicianas (Guardia Nacional) y regulares (Ejército de Línea) hasta entonces en el servicio de fronteras, hacia esos conflictos. Es en ese contexto de relaciones de fuerza y de dependencia estatal con respecto a los «indios amigos» en el que hay que analizar las experiencias indígenas asociadas al acceso a la propiedad de la tierra en jurisdicción bonaerense ${ }^{16}$.

De hecho, los titulares de las fracciones que subdividieron La Barrancosa en su mayoría estuvieron asociados al servicio de armas en órbita estatal. Esa fue una de las principales fuentes de legitimación a la que recurrieron los caciques para reclamar con éxito la donación ${ }^{17}$. Desde la década de 1840 y especialmente en las dos décadas siguientes, un cuerpo de hombres al mando de los caciques Martín Collinao y Pedro Melinao había servido militarmente en la frontera de Bragado y Nueve de Julio incorporados a la administración militar del Estado ${ }^{18}$. Este fue uno de los grupos que participó en la trama de relaciones políticas y económicas del Negocio Pacífico de Indios desde la primera mitad del siglo XIX. ${ }^{19}$ Ambos caciques, según Hux, eran hijos de Luis Melipan - muerto en 1828-, líder patriota durante la guerra que siguió al pronunciamiento de la independencia al oeste de los Andes - denominada Guerra a Muerte - (ver cuadro 1) ${ }^{20}$.

15 En cambio, en el ámbito periurbano, existió un antecedente en la década de 1850 con la donación a la tribu de Maycá, origen de Villa Fidelidad (Lanteri et al., 2011. Barbuto, 2014. Lanteri y Pedrotta, 2018).

16 De Jong, 2015.

17 Literas, 2016.

18 Hux, 2004 [1992]. Se ha demostrado que la condición de «indio amigo» no fue sinónimo de subordinación a la esfera estatal (de Jong, 2008) ni que la militarización conllevó disciplina o menos aún control capilar sobre los ritmos y las condiciones de vida indígenas.

19 Ratto, 2003: 191-222.

20 Hux, 2007. Villar y Jiménez, 2011. 


\section{CuAdro 1. Parentesco De los PRincipales Líderes}

DE LA POBLACIÓN INDÍGENA DE LA BARRANCOSA

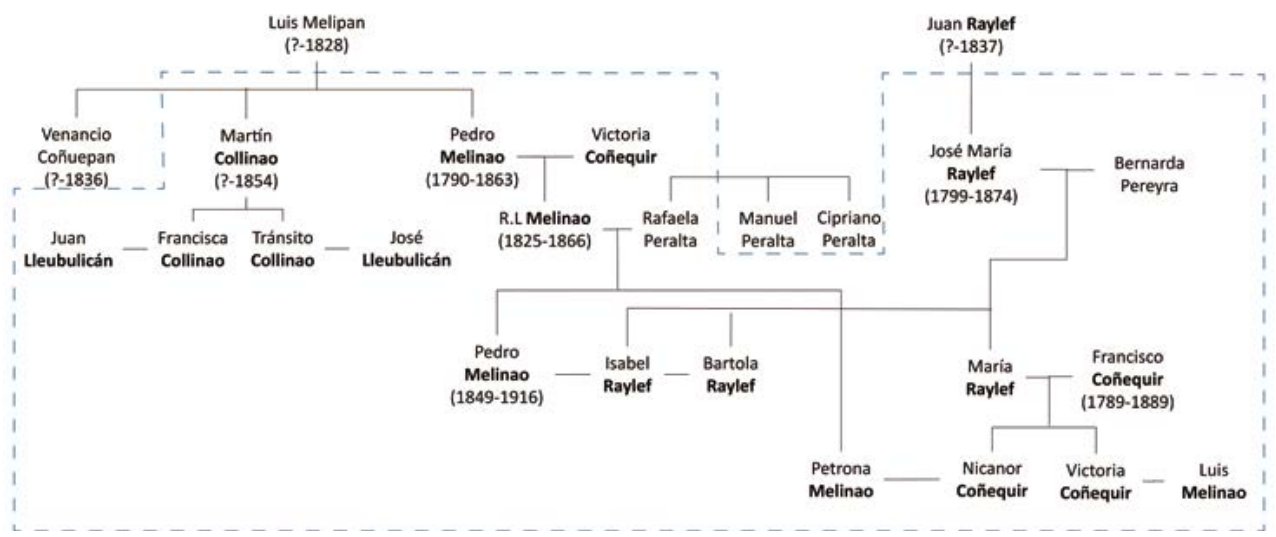

Fuente: elaboración propia en base a Hux (2004 [1992]). AHMB, Registro de Vecindad, 18901891. Censo Nacional de 1895. Se identifica en línea de puntos a quienes habitaron La Barrancosa. En los casos en que se desconoce a la mujer solo figura el hombre (por ejemplo, Luis Melipan). No se presenta toda la descendencia de cada unión conyugal sino la más significativa por motivos de espacios y a efectos de reconstruir el parentesco entre las principales familias (por ejemplo José María Raylef).

Además de la militar, la otra dimensión para abordar el acceso y uso de la tierra indígena es el parentesco. En este caso la antropología histórica de las Pampas y Norpatagonia cuenta con el abordaje pionero de Martha Bechis $^{21}$, quien identificó un sistema de casamientos entre primos cruzados patri-laterales que unía y cohesionaba a los linajes políticamente más importantes de la tribu, los Melinao y Raylef, conservando así el equilibrio de poder. Ambos grupos intercambiaban alternativamente mujeres - similar al modelo levi-straussiano de intercambio discontinuo - sin importar tanto a qué generación pertenecían, sino que ello garantizase la reciprocidad a modo de pacto entre iguales, protegiendo a la descendencia y consolidando la parcialidad en un contexto de migración y asentamiento bajo jurisdicción estatal —algo característico de la condición de «indio amigo»- Nuestra propia reconstrucción del parentesco apoya estas afirmaciones. Es más, sobre este intercambio se dio no solo la sucesión de los cacicazgos de los Melinao y Raylef sino también tuvo contacto con la organización de las prestaciones militares y con la distribución de una parte significativa de La Barrancosa. Siguiendo a Hux ${ }^{22}$,

\footnotetext{
21 Bechis, 2010 [1990].

22 Hux, 2004 [1992].
} 
ambos caciques estaban emparentados al menos por vía matrimonial con los Coñequir y Lleubulicán, familias que dieron líderes y hombres de lanza de modo prolongado y numeroso: Pedro Melinao al unirse conyugalmente con Victoria Coñequir — pariente del capitanejo Francisco Coñequir-; Martín Collinao a través de la unión de sus hijas Francisca y Tránsito con el cacique Juan Lleubilicán y el que probablemente fue su hermano José. A la muerte del cacique Collinao en 1854 y de Melinao en 1863 le sucedió el liderazgo de Ramón Luis Melinao y José María Raylef (ver cuadro 2) ${ }^{23}$.

El primero, Ramón Luis Melinao, era hijo de Pedro Melinao y Victoria Coñequir y según Hux se unió conyugalmente con Rafaela Peralta, hermana de Manuel y Cipriano Peralta, capitanejos de Cipriano Catriel en la zona de Azul y Tapalqué donde, por otro lado, la gente de Melinao había estado asentada antes de trasladarse a La Barrancosa. Primero alférez (1858) y después teniente (1858-1863) ascendió a ayudante mayor de Línea tras la muerte de su padre (1863-1866). De modo similar a otros «indios amigos» como los Coliqueo, Rondeau y Tripailaf los hijos de los líderes políticos fueron incorporados a las jerarquías del Ejército de Línea e incluso a la Plana Mayor de la administración militar fronteriza y asistieron a sus padres como escribientes, secretarios y/o lenguaraces. Esto ha sido explicado en términos de incorporación selectiva digitada por el Estado argentino en virtud de reforzar y reproducir ciertos liderazgos indígenas ${ }^{24}$. También, sin embargo, puede ser entendido como una estrategia subalterna para mejorar las condiciones de relacionamiento e inserción social en el marco de la organización y construcción del Estado, en un contexto cambiante de relaciones de fuerza, a partir de la apropiación y ejercicio de capitales y recursos asociados a la administración militar.

El segundo, José María Raylef, era hijo de Juan Raylef, otro líder patriota en la Guerra a Muerte, fallecido en 1837. Tras la muerte de Melinao, José María Raylef dejó de ser considerado caciquillo en los registros asociados a la administración militar, para hacerlo como cacique. Según Hux, de hecho, era el principal líder de La Barrancosa a ojos de las autoridades fronterizas. Lo que puede confirmarse es que a través de sus hijas reforzó el parentesco con los Melinao y Coñequir: Bartola e Isabel Raylef se casaron con Pedro Melinao, hijo de Ramón Luis Melinao y nieto del primer cacique Pedro Melinao mientras que María Raylef lo hizo con el capitanejo Francisco Coñequir.

${ }^{23}$ Los hechos y factores involucrados en la sucesión cacical tras la muerte de Pedro Melinao han sido ampliamente tratados y explicados en virtud de factores parentales (Bechis, 2010 [1990]) y de la incidencia de la política estatal (Hux, 2004 [1992]). Este no es el lugar para abordar dicho problema por tanto remitimos a los trabajos citados.

${ }^{24}$ Quijada, 2011. 
Como veremos en la reconstrucción de las unidades domésticas que habitaron La Barrancosa, este entrecruzamiento parental entre familias vinculadas al liderazgo político-militar continuó en las siguientes generaciones.

\section{Cuadro 2. Caciques y Capitanejos de La Barrancosa}

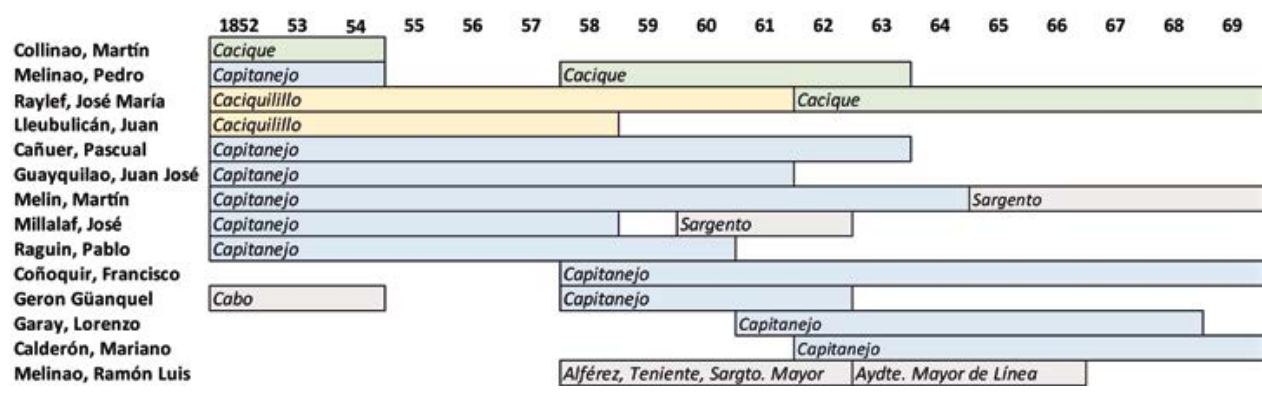

Fuente: elaboración propia en base a listas de revista del AGN y el SHE.

Ahora bien, tal como se adelantó, la militarización y el parentesco indígena aquí rápidamente reseñados estuvieron asociados al acceso y el uso de la tierra. Esto es demostrado principalmente por la fuerte correlación entre las experiencias políticas y militares, las conexiones sociales y la distribución de tierras (ver cuadro 3). Una primera observación al respecto es que los descendientes de los caciques Pedro Melinao y José María Raylef, así como de los capitanejos Francisco Coñequir y Pascual Cañiú concentraron a lo largo del siglo XIX la mayor cantidad de tierras donadas a la tribu en 1863.

Del mismo modo, empero, debe mencionarse que no todos los individuos relevados en las listas de revista y de racionamiento, así como tampoco sus familias, aparecen en los registros asociados a La Barrancosa. Aún no disponemos de información suficiente para proponer afirmaciones concluyentes al respecto. En cambio, existen indicios que sugieren tendencias simultáneas de desarticulación político-étnica «tribal»e inserción laboral proletarizada en otros establecimientos rurales y urbanos. Sobre todo, en la medida en que a lo largo de las últimas décadas del siglo XIX y especialmente tras la Conquista del Desierto, se consolidó la forma política estatal y el capitalismo en las 1lanuras pampeanas ${ }^{25}$.

No obstante, como se ha adelantado, a la distribución de derechos de titularidad con respecto a las fracciones de tierras - eso que muestran las mensuras-

25 Salomón Tarquini, 2010. Salomón Tarquini, Rollhauser y Nagy, 2014. 


\section{CuAdro 3. Titulares de las tierRas EN 1906 y RElaCióN CON EL SERVICIO DE ARMAS DE LA TRIBU DE MELINAO}

\begin{tabular}{|c|c|c|c|}
\hline & Titular & Has. & \\
\hline \multirow{4}{*}{ MELINAO } & Pedro Melinao & 828.50 & $\begin{array}{l}\text { Hijo del cacique Ramón Luis Melinao. Soldado (1858-1867), teniente (1874) } \\
\text { y cacique }\end{array}$ \\
\hline & Petrona Melinao de Coñequir & 56.33 & Hija del cacique Ramón Luis Melinao y esposa de Nicanor Coñequir \\
\hline & Silverio Melinao & 26.51 & \multirow{2}{*}{ Parientes del cacique Pedro Melinao } \\
\hline & Luis Melinao & 26.51 & \\
\hline \multirow{13}{*}{ RAYLEF } & Bartola Railef de Melinao & 41.42 & \multirow{2}{*}{ Esposas del cacique Pedro Melinao e hijas del cacique José María Raylef } \\
\hline & Isabel Raylef & 39.76 & \\
\hline & Cayetano Raylef & 39.76 & Alférez $(1864-1869,1874)$ e hijo del cacique José María Raylef \\
\hline & Visitación Raylef & 33.02 & Hijos/as y parientes del cacique José María Raylef \\
\hline & Felipe Raylef & 39.76 & \\
\hline & José María Raylef & 39.76 & \\
\hline & Ramón Raylef & 41.42 & \\
\hline & Anastacio Raylef & 41.42 & \\
\hline & María Raylef & 41.42 & \\
\hline & Justina Raylef & 41.42 & \\
\hline & Benigno Raylef & 39.76 & \\
\hline & Germán Raylef & 39.76 & \\
\hline & Bernarda Pereyra de Raylef & 41.42 & \\
\hline OLGUÍN & Justa Olguín de López & 35.04 & Esposa de Cayetano Raylef \\
\hline \multirow{7}{*}{ COÑEQUIR } & Pascual Coñequir & 331.40 & \multirow{7}{*}{$\begin{array}{l}\text { Hijos/as de Francisco Coñequir, soldado (...-1854) y capitanejo (1858-1869), y } \\
\text { de María Raylef, hija del cacique José María Raylef }\end{array}$} \\
\hline & Nicanor Coñequir & 46.39 & \\
\hline & Silvano Coñequir & 46.39 & \\
\hline & Abdón Coñequir & 46.39 & \\
\hline & Martina Coñequir de Martínez & 46.39 & \\
\hline & Angela Coñequir & 46.39 & \\
\hline & Victoria Coñequir & 46.39 & \\
\hline \multirow{5}{*}{ SÁNCHEZ } & Mariano Sánchez & 381.11 & $\begin{array}{l}\text { Soldado (1874) y probablemente hijo de Dionisio Sánchez, soldado } \\
(1858-1867)\end{array}$ \\
\hline & Flora Sánchez & 66.28 & \multirow{4}{*}{ Parientes de Dionisio Sánchez, soldado (1858-1867) } \\
\hline & Juan de Dios Sánchez & 66.28 & \\
\hline & Marcos Sánchez & 66.28 & \\
\hline & Felisa Sánchez & 66.28 & \\
\hline \multirow{9}{*}{$\begin{array}{l}\text { NAHUEL Y } \\
\text { CURUMÁN }\end{array}$} & Alberto Nahuel y Curumán & 36.45 & \multirow{9}{*}{ Probablemente parientes de Gabriel Nahuel, sargento (1864-1869) } \\
\hline & Francisco Nahuel y Curumán & 36.45 & \\
\hline & Juana Nahuel y Curumán & 36.45 & \\
\hline & Rosa Nahuel y Curumán & 36.45 & \\
\hline & Antonia Nahuel y Curumán & 36.45 & \\
\hline & Gerónimo Nahuel y Curumán & 36.45 & \\
\hline & Wenceslao Nahuel y Curumán & 36.45 & \\
\hline & Josefa Curuman Railef de Nahuel & 36.45 & \\
\hline & Tomás Nahuel y Curumán & 36.45 & \\
\hline
\end{tabular}




\begin{tabular}{|c|c|c|c|}
\hline & Titular & Has. & \\
\hline \multirow{7}{*}{ CAÑIÚ } & Martiniano Cañiú & 54.68 & \multirow{7}{*}{ Parientes de Pascual Cañiú, capitanejo (...-1863) y su esposa Andrea Ancalao } \\
\hline & Manuela Cañiú & 54.68 & \\
\hline & Antonia Cañiú & 54.68 & \\
\hline & Césarea Cañiú & 54.68 & \\
\hline & Sebastiana Cañiu & 53.02 & \\
\hline & Juan Cañiú & 26.51 & \\
\hline & Severa Cañiú & 26.51 & \\
\hline \multirow{2}{*}{ BARRANCOS } & Martín Barrancos & 248.87 & Ocupante reconocido como "miembro de la tribu" en la declaración de 1889 \\
\hline & Angel Barrancos & 36.97 & Labrador, casado con Martina Cristóbal \\
\hline \multirow{4}{*}{ GUAYQUILAO } & Noguera Guayquilao & 166.81 & $\begin{array}{l}\text { Soldado (1864-1869), sargento (1874) y probablemente parientes de Juan José } \\
\text { y José María Guayquilao }\end{array}$ \\
\hline & Margarita Guayquilao Díaz & 53.02 & \multirow{2}{*}{$\begin{array}{l}\text { Probablemente parientes de Juan José Guayquilao, capitanejo }(\ldots-1861) \text { y } \\
\text { José María Guayquilao, sargento (1864-1868) }\end{array}$} \\
\hline & Margarita Guayquilao de Cristóbal & 29.93 & \\
\hline & Juan José Guayquilao & 13.25 & $\begin{array}{l}\text { Soldado (1864-1865), cabo (1865-1868) y probablemente parientes de Juan } \\
\text { José y José María Guayquilao }\end{array}$ \\
\hline \multirow{2}{*}{ ALONSO } & Florentino Alonso & 82.84 & \multirow{2}{*}{$\begin{array}{l}\text { Probablemente parientes de Alonso } 1^{\circ} \text {, soldado (...-1854), Ceferino Alonso, } \\
\text { soldado (1858-1869) y/o Manuel Alonso, cabo (1858-1864), }\end{array}$} \\
\hline & Severa Alonso & 26.51 & \\
\hline \multirow[b]{2}{*}{ COLLINAO } & Tránsito Collinao de Llublicán & 41.42 & Hija del cacique Martín Collinao (...-1854) \\
\hline & María Collinao & 41.42 & $\begin{array}{l}\text { Probablemente pariente del cacique Martín Collinao, Martín Collinao, soldado } \\
\text { (1858-1863) y/o Pedro Collinao, soldado (1852-1854) }\end{array}$ \\
\hline ESCALADA & Pedro Escalada & 82.85 & Soldado $(1864-1869,1874)$ \\
\hline \multirow[b]{2}{*}{ COLIQUEO } & Eufemio Coliqueo & 27.34 & \multirow{2}{*}{$\begin{array}{l}\text { Probablemente parientes de Antonio Coliqueo, soldado }(\ldots-1854) \text { y sargento } \\
\text { (1858), Manuel Coliqueo, sargento }(1860-1869) \text { y/o Pedro Coliqueo, soldado } \\
(1866-1869,1874)\end{array}$} \\
\hline & Felipe Coliqueo & 26.51 & \\
\hline CAYUNAO & Marcos Cayunao & 66.28 & Soldado (1864-1869) \\
\hline \multirow[b]{2}{*}{ SOTELO } & Martín Sotelo & 26.51 & Soldado (1861-1863) y pariente de Mariano Sotelo, soldado $(1854-1869,1874)$ \\
\hline & Dominga Sotelo & 26.51 & $\begin{array}{l}\text { Pariente de Mariano Sotelo, soldado }(1854-1869,1874) \text {, probablemente casada } \\
\text { con Pantaleón Cañiulef, soldado }(1861-1869,1874)\end{array}$ \\
\hline \multirow{3}{*}{ LLUBLICÁN } & Rita Llublican & 26.51 & \multirow{3}{*}{$\begin{array}{l}\text { Probablemente parientes del cacique Juan Lleubul (..-1858), Mariano } \\
\text { Lleubulicán, soldado (1868), Juan Lleubulicán } 1^{\circ} \text {, soldado (1860-1862), Juan } \\
\text { Lleubilicán } 2^{\circ} \text {, soldado (1861-1864) y cabo }(1874) \text {, José Lleubulicán, soldado } \\
(1864-1869,1874) \text { y/o Ignacio Lleubulicán }(1874)\end{array}$} \\
\hline & Félix Llublican & 13.25 & \\
\hline & Teresa Palusa de Llublicán & 13.25 & \\
\hline \multirow{2}{*}{ TATALAO } & Andrés Tatalao & 23.19 & Soldado (...-1868) y cabo (1868-1869) \\
\hline & Máxima P. de Tatalao & 13.25 & Pariente de Andrés Tatalao \\
\hline NEHIPÁN & Andrés Nehipán & 27.34 & \\
\hline CRISTÓBAL & Juana Pio de Cristóbal & 26.51 & \\
\hline MELÍN & Genara Melín & 26.51 & $\begin{array}{l}\text { Probablemente pariente de Martín Melín, capitanejo (...-1865) Lorenzo Melín, } \\
\text { soldado (1860-1867) }\end{array}$ \\
\hline HUICHÚ & Lorenzo Huichu & 26.61 & \\
\hline GARCÍA & Martín Zoilo García & 26.51 & \\
\hline ALMIRÓN & Margarita Almirón & 26.51 & Probablemente pariente de Juan Almirón, soldado $(1858-1867,1874)$ \\
\hline MANQUEHUAL & Basilia Manquehual & 13.25 & \\
\hline \multirow[t]{2}{*}{ LARREA } & José A. Larrea & 11.46 & \\
\hline & & 4672.96 & \\
\hline
\end{tabular}

Fuente: elaboración propia en base a ADGPBA, Bragado, $\mathrm{n}^{\circ}$ 98, Pedro Melinao y otros, 1903 y $\mathrm{n}^{\circ}$ 100; Pedro Melinao y otros, 1906. AHMB, Registro de Vecindad, 1890-1891. AGN, Sala III, Listas de Revista; SHE, Listas de Revista. Hux (2004 [1992]; 2007). 
se le superpone un escenario diferente de ocupación y uso. En efecto, como se verá, este relevamiento de ocupaciones en La Barrancosa dista mucho de identificar con precisión y menos aún con exhaustividad a la población que ocupó y utilizó esas tierras. A continuación, se presenta un plano de la tribu de Melinao según las mesuras de 1903 y 1906 discriminando los propietarios de los lotes y las unidades de población registradas en el relevamiento catastral (ver mapa 2).

\section{MaPA 2. Titulares y OCUPANTES DE LAS TIERRAS DE LA TRIBU}

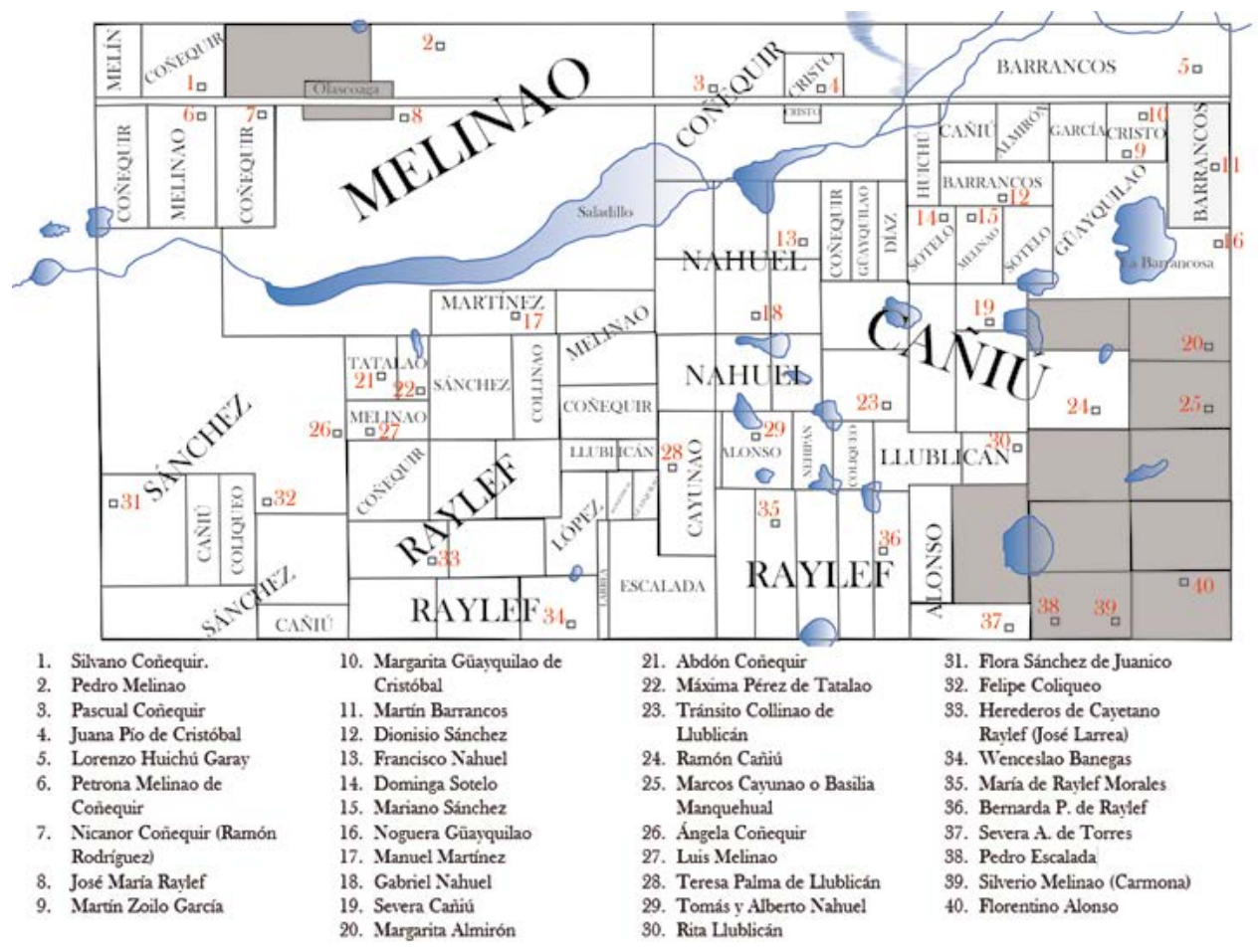

Fuente: elaboración propia en base a ADGPBA, Bragado, n 98, Pedro Melinao y otros, 1903 y $\mathrm{n}^{\circ} 100 ;$ Pedro Melinao y otros, 1906. AHMB, Registro de Vecindad, 1890-1891. En gris las tierras expropiadas a la tribu en virtud de construir la estación del ferrocarril y la traza urbana de Olascoaga, así como los campos para extender el ejido de Bragado.

Hasta aquí una primera reconstrucción del contexto en que tuvo lugar la donación de tierras a la población indígena de Bragado, sus conexiones con el servicio de armas, la incidencia del parentesco y la identificación de sus 
titulares. Sin embargo, huelga insistir, esto dice poco sobre la ocupación y el uso de La Barrancosa, principal objeto de este trabajo.

\section{OCUPACIÓN Y USO DE LA BARRANCOSA}

Las «tierras de la tribu» y «La Barrancosa» son las formas genéricas para denominar e identificar a las aproximadamente cinco mil hectáreas donadas al cacique Pedro Melinao y su gente en 1863. Es un rectángulo trazado en dirección noreste-sudoeste a lo largo del río Saladillo, salpicado por pequeñas lagunas entre la que destaca La Barrancosa en su sección más oriental (ver figura 1). Este río es tributario de la laguna del Bragado, inmediata al ejido del pueblo homónimo, espejo de agua más importante en esa región al sur del

Figura 1. El Río SALAdillo a SU Paso POR LAS TIERRAS DE LA TRIBU DE MELINaO

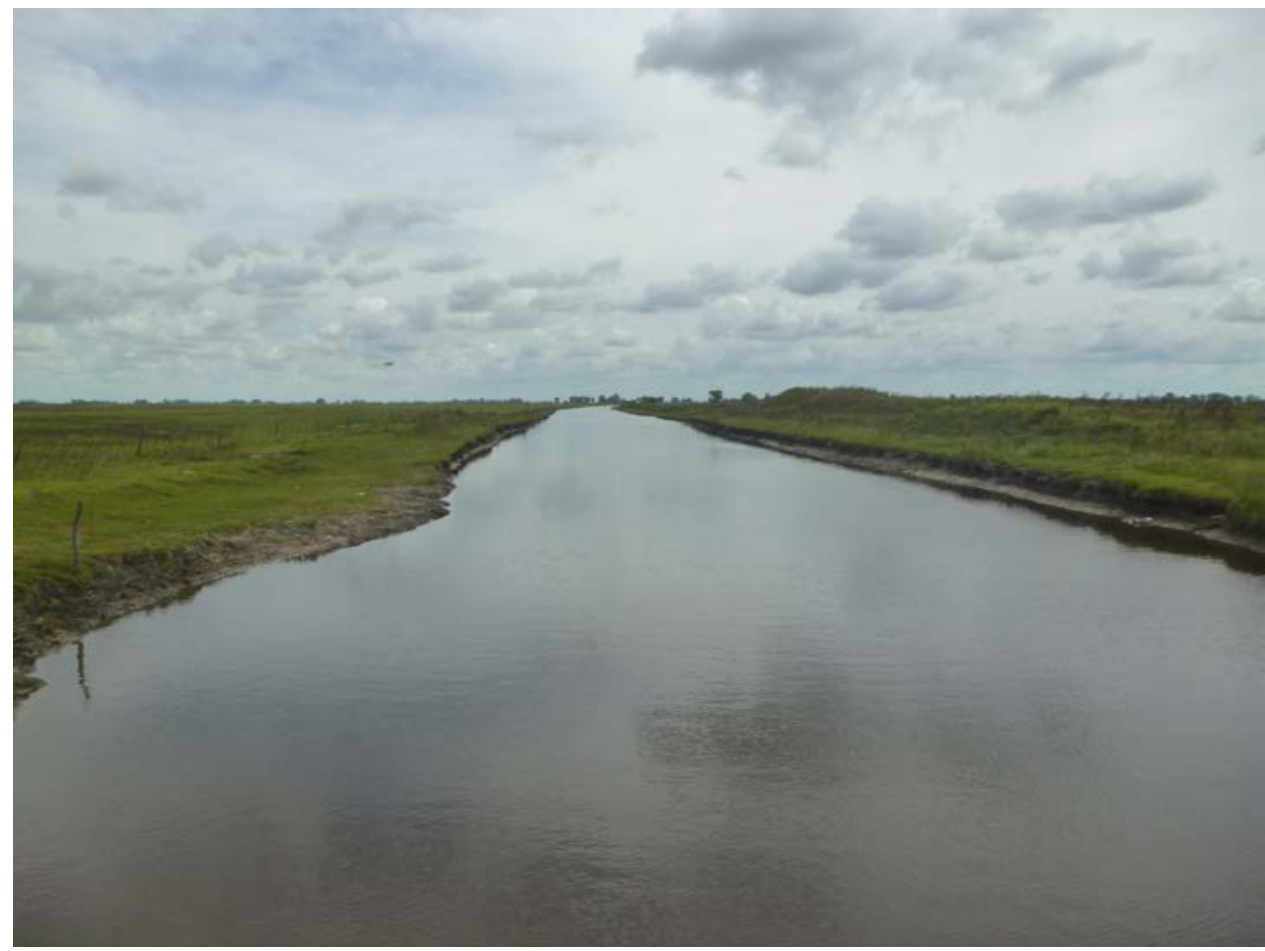

Fuente: fotografía del autor en dirección noreste-sudoeste. 
río Salado y con el que se conecta a través del canal República de Italia construido en la década de 1980. En términos generales es una zona baja, cuyas condiciones agronómicas son más propias para la ganadería; algo que contrasta con el uso actual dedicado en gran parte a la agricultura de soja, la más difundida en las llanuras pampeanas.

\section{Las grandes familias: Melinao, Raylef, Coñequir}

Hubo tres familias de la tribu dedicadas al liderazgo político, la dirección militar y posteriormente a la administración y gestión de La Barrancosa: Melinao, Raylef y Coñequir. Como se ha visto, ellas estuvieron unidas por múltiples lazos parentales en generaciones sucesivas (cuadro 1) ${ }^{26}$. Los reclamos y el litigio que dieron lugar a la donación de esas tierras $(1859-1863)^{27}$, así como las exigencias y la obtención del licenciamiento del servicio de armas al Estado (1869) ${ }^{28}$ son hitos de dicho liderazgo.

Las fracciones registradas a título de Pedro Melinao en particular y de los Melinao en general representan la mayor cantidad de tierras, un quinto del total. Aquí sin embargo hay que distinguir las «chacras» y los «puestos», las dos nominaciones catastrales que tuvieron. La primera alude con toda seguridad a una fracción menor del total de tierras mensuradas a nombre de Melinao, en el margen occidental de las vías del ferrocarril, donde el cacique encabezaba la única unidad doméstica ahí relevada (mapa 3, fracción A, posición 1) y a un lote donde fue registrada la población de Luis Melinao (mapa 3 , fracción $\mathrm{B}$, posición 2), quien según Hux era hermano del cacique. La segunda denominación referenció a un variado conjunto de asentamientos (puestos) en el terreno más amplio, en torno al curso del Saladillo (mapa 3, fracción C). Pedro Melinao, criador y labrador, estuvo casado con Isabel Raylef y Bartola Raylef — quien según Hux no podía tener hijos, algo que los registros demográficos sugieren-. Muy probablemente fue a través de estos matrimonios con las hijas de José María Raylef - de quien a su vez era sobrino- que Melinao adquirió la titularidad de parte de las tierras que aquel había ocupado (mapa 3, fracción C, posición 3). En efecto para 1903 ambas mujeres eran adjudicatarias de tierras a título de José María Raylef.

26 No se discrimina aquí el parentesco «biológico» o «político». El compadrazgo y la adopción bilateral son prácticas constitutivas del parentesco. Los avances realizados con respecto al parentesco indígena refuerzan esta decisión (Hux, 2007. Villar y Jiménez, 2011. Davies, 2013).

27 Literas, 2016.

28 Hux, 2004 [1992]. 
Con respecto a las chacras de Melinao (mapa 3, fracciones A y B) deben mencionarse algunas cuestiones. En primer lugar, la existencia de matrimonios de hombres Melinao con mujeres de las otras dos principales familias de la tribu: Raylef y Coñequir (ver cuadro 4). En segundo lugar, que es posible imaginar una cierta autonomía con respecto al trabajo y la tierra. Los hombres adultos ejercían el pastoreo de ganado y la agricultura. No hay registros de mano de obra empleada de modo estable ni tampoco de que miembros de

\section{Mapa 3. Titulares y ocupantes de las tierRas De La tRIBU (SElección)}

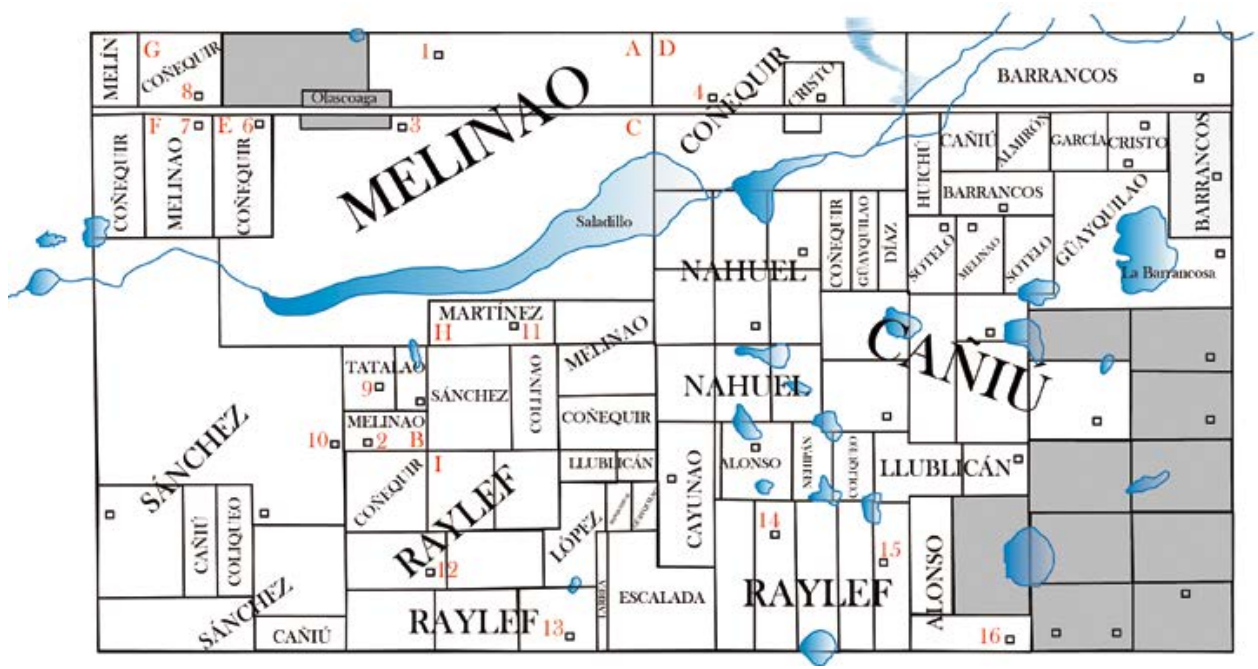

Fuente: elaboración propia en base a ADGPBA, Bragado, $\mathrm{n}^{\circ}$ 98, Pedro Melinao y otros, 1903 $\mathrm{y} \mathrm{n}^{\circ} 100 ;$ Pedro Melinao y otros, 1906. AHMB, Registro de Vecindad, 1890-1891.

\section{Cuadro 4. Población de las Chacras de Pedro Melinao}
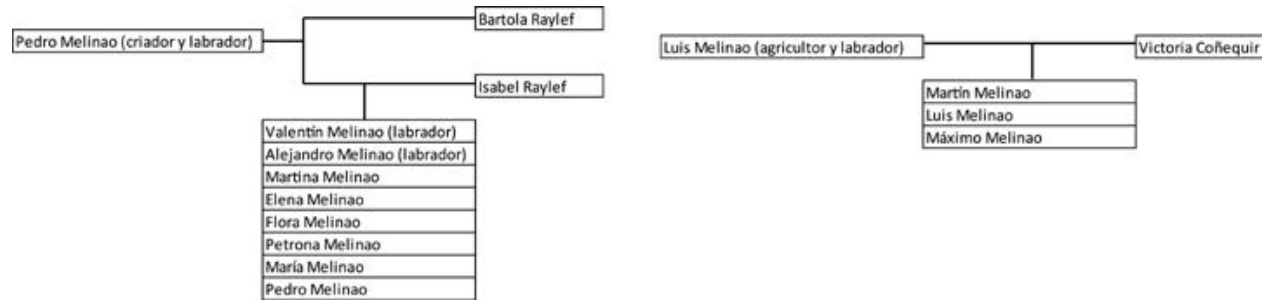

Fuente: elaboración propia en base a ADGPBA, Bragado, $n^{\circ}$ 98, Pedro Melinao y otros, 1903 y $\mathrm{n}^{\circ} 100$; Pedro Melinao y otros, 1906. AHMB, Registro de Vecindad, 1890-1891. Censo Nacional de 1895. 
estas unidades, mujeres u hombres, se emplearan en otros lugares. Esto pudo corresponderse con un mayor control sobre el acceso y uso de la tierra. Por último, hay que añadir que ambas fracciones fueron mensuradas efectivamente a título de Pedro Melinao y Luis Melinao. Es decir, el parentesco fue origen de derechos de propiedad.

El panorama de los puestos de Melinao dista de lo que se observa en las chacras. Con toda seguridad, tal como se adelantó, estos se dispersaron a lo largo del extenso campo en torno al trayecto más ancho del Saladillo, tierras bajas, bañados en algunos casos (mapa 3, fracción C), separados de la chacra por el tendido ferroviario que une Bragado y Olascoaga. En una primera aproximación nos preguntamos sobre el uso que podría tener esta fracción, la más amplia por lejos de todas las tierras de la tribu y sobre la cual los registros catastrales ofrecen menos información ${ }^{29}$ (ver figura 2).

Según los relevamientos de tierras allí habría habitado el cacique José María Raylef y de hecho, como se adelantó, fue el matrimonio de Pedro Melinao con dos de sus hijas (Bartola e Isabel) lo que habilitó que esas extensiones fueran de su titularidad. Empero, el poblamiento de esta amplia y agronómicamente más pobre fracción contrasta con lo que se verá frecuentemente en el resto. Aquí predomina un heterogéneo conjunto de unidades domésticas de origen indígena, criollo e incluso inmigrante europeo, dedicado por entero - salvo un caso- a ser jornaleros. En efecto esa es la definición de «puesto»: emplazamiento en función de controlar la cría de ganado y los derechos de uso del espacio, al que le pueden subyacer formas de medianería de la explotación con el titular, en este caso los Melinao y Raylef. Una especie de sub-arrendamiento mediante renta de trabajo que, no obstante, en el marco de la vaguedad de los derechos de propiedad y transmisión de las tierras de «indios amigos» pudo dar lugar al pedido de títulos por posesión treintañal.

El primer conjunto de casos son unidades indígenas (ver cuadro 5). Una conformada por Bartola Raylef (esposa sin hijos de Pedro Melinao), Silverio Melinao y un matrimonio Peralta y otra por los Coliqueo - otra familia indígena de extensa trayectoria en la frontera-. En ellas, todos los hombres adultos eran jornaleros. Esto sugiere varias observaciones. Por un lado, el hecho de que nadie fuera reconocido como hacendado, criador de ganado ni labrador - categorías asociadas a la propiedad - apoya la idea de medianería o sub-arrendamiento de estas tierras. Por otro lado, la presencia de los Coliqueo confirma la existencia de sólidos y duraderos lazos al interior del mun-

29 Literas, 2016. 
do indígena que trascendieron ampliamente las circunscripciones nominales «tribales», problema de estudio sobre el que aún resta mucho avanzar.

Un segundo conjunto de casos fueron unidades mixtas, donde primó el matrimonio con mujeres indígenas de las familias Raylef, Alonso y quizás Cristóbal (ver cuadro 6) ${ }^{30}$. Aquí la principal observación es que la unión conyugal mixta fue condición necesaria para que un no indígena pudiera habitar y explotar la tierra. Esto es un dato relevante, observado en otras tribus ${ }^{31}$, ya que como se verá más adelante fue el primer paso de la posterior enajenación de tierras indígenas.

Un tercer y último conjunto consiste en puestos poblados enteramente por no indígenas (ver cuadro 7). Esos fueron los casos de las familias españolas Jáuregui e Iturbea y las argentinas Juanico y Requelme, todas dedicadas a la cría de ganado. La singularidad aquí es que no hubo miembros de la tribu; que compartían puesto más de una unidad matrimonial —en el caso de los Juanico por ampliación matrilocal-; y que a diferencia del resto no fueron relevados en el censo que se practicó pocos años después. Se desconoce aún el origen y tipo de relaciones que subyació a este hecho, aunque debió atribuirse al sub-arrendamiento, forma al uso en el resto de establecimientos de la campaña bonaerense.

\section{Cuadro 5. Población de los Puestos de Pedro Melinao (1)}

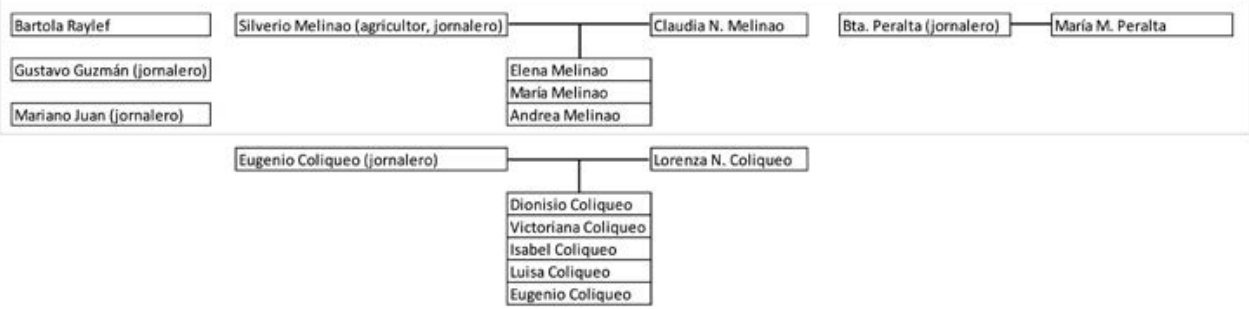

Fuente: elaboración propia en base a ADGPBA, Bragado, $\mathrm{n}^{\circ}$ 98, Pedro Melinao y otros, 1903 y $\mathrm{n}^{\circ} 100$; Pedro Melinao y otros, 1906. AHMB, Registro de Vecindad, 1890-1891. Censo Nacional de 1895. En línea continua se identifican las unidades domésticas.

30 En algunos casos, también, a estas familias se agregaron otras personas.

31 Literas, 2015. 


\section{Cuadro 6. Población de Puestos de Pedro Melinao (2)}
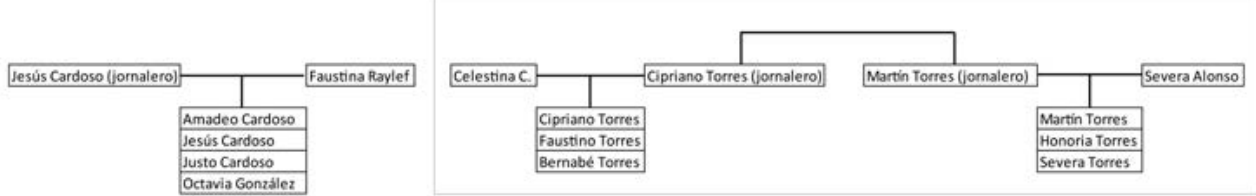

Fuente: elaboración propia en base a ADGPBA, Bragado, n ${ }^{\circ}$ 98, Pedro Melinao y otros, 1903 $\mathrm{y} \mathrm{n}^{\circ} 100$; Pedro Melinao y otros, 1906. AHMB, Registro de Vecindad, 1890-1891. Censo Nacional de 1895. En línea continua se identifican las unidades domésticas.

\section{Cuadro 7. Población de los Puestos de Pedro Melinao (3)}

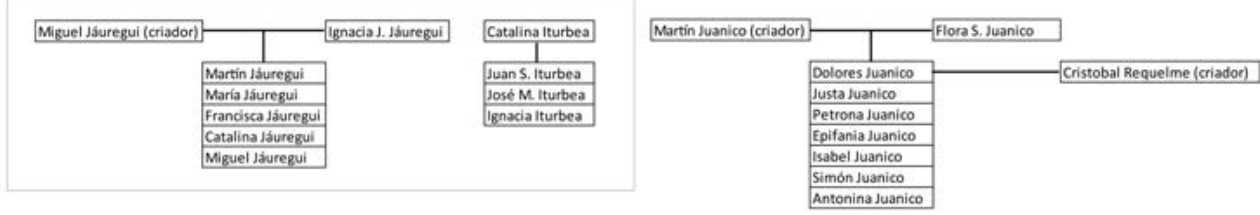

Fuente: elaboración propia en base a ADGPBA, Bragado, $\mathrm{n}^{\circ}$ 98, Pedro Melinao y otros, 1903 y $\mathrm{n}^{\circ} 100$; Pedro Melinao y otros, 1906. AHMB, Registro de Vecindad, 1890-1891. Censo Nacional de 1895. En línea continua se identifican las unidades domésticas.

\section{Figura 2. Los Puestos de Melinao}

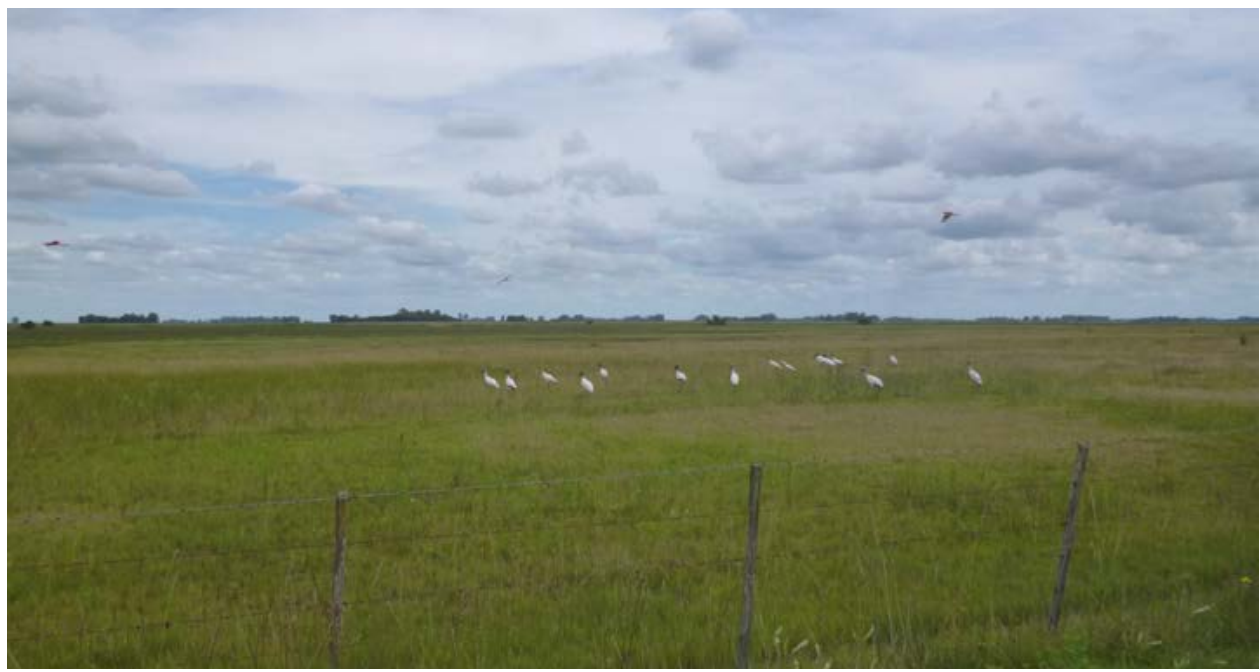

Fuente: fotografía del autor. 
Coñequir fue otra familia con gran cantidad de tierras, más específicamente del capitanejo Francisco Coñequir - lugarteniente de Pedro Melinao, concuñado del nieto de este y yerno de José María Raylef - y de sus descendientes. Cuatro chacras fueron relevadas a fines de siglo XIX a título de los Coñequir, que reunían un total de más de seiscientas hectáreas: las de Pascual, Nicanor, Silvano y María (ver cuadro 8). Ellas se entrelazan con las de Melinao ocupando la mayor parte de la parte oeste de La Barrancosa. Todas, sin excepción, estuvieron ubicadas contiguo a ellas, como una suerte de continuidad.

La primera (mapa 3, fracción D) tenía similares características que la chacra principal de Pedro Melinao. Separada del resto de los campos de La Barrancosa por la vía férrea que une Bragado y Olascoaga, en la zona más alta, se extendía hacia el este a orillas del Saladillo. Era la tercera fracción más grande de tierras de toda la tribu. Allí vivía Pascual Coñequir dedicado a la cría de ganado, con su esposa e hijos (mapa 3, posición 4). Por los registros vecinales se desconoce si esta chacra era habitada por más personas.

Las chacras de Nicanor y Silvano Coñequir se ubicaban en el otro margen, al oeste de las chacras de Melinao. El primero estaba casado con Petrona Melinao, hija de Ramón Luis Melinao y hermana de Pedro Melinao (mapa 3, fracción $\mathrm{E}$, posición 6) y precisamente ella tenía también derechos sobre una fracción aledaña (mapa 3, fracción $\mathrm{F}$, posición 7). El segundo estaba casado con María Rosa de la que se desconoce más información (mapa 3, fracción G, posición 8). La última chacra era la de María Raylef, hija del cacique José María Raylef y viuda del capitanejo Francisco Coñequir. Muy probablemente se ubicaba al sudeste de las chacras de Melinao, cerrando círculo de ocupaciones de Coñequir sobre estas (mapa 3, posiciones 9 y 10). En esa zona también estaba ubicado el puesto del español Manuel Martínez (mapa 3, fracción $\mathrm{H}$, posición 11), casado con Martina Coñequir, una relación de similares características que las que se observaron en los puestos de Melinao. Huelga insistir, en muchos casos esa fue la condición de la futura apropiación no indígena de La Barrancosa por vía matrimonial.

A diferencia de los Melinao y Coñequir, solo tenemos constancia de una chacra a nombre de Raylef, la de Cayetano (mapa 3, fracción I, posición 12). En efecto el caso de los Raylef es donde se observan mayores discrepancias y contrastes entre los registros militares y vecinales, los relevamientos de ocupaciones y las mensuras de tierras. Si a esto le sumamos que en 1895 solo tres personas fueron censadas con el apellido Raylef en todo el cuartel séptimo de Bragado, tenemos el cuadro completo del problema con respecto a cómo se diluyó en las fuentes el apellido de uno de los linajes de trayectoria más relevante en la política indígena de las Pampas, tal como han mostrado los estudios referenciados de Hux y Bechis. 


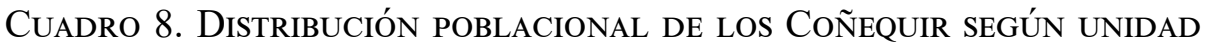
DOMÉSTICA

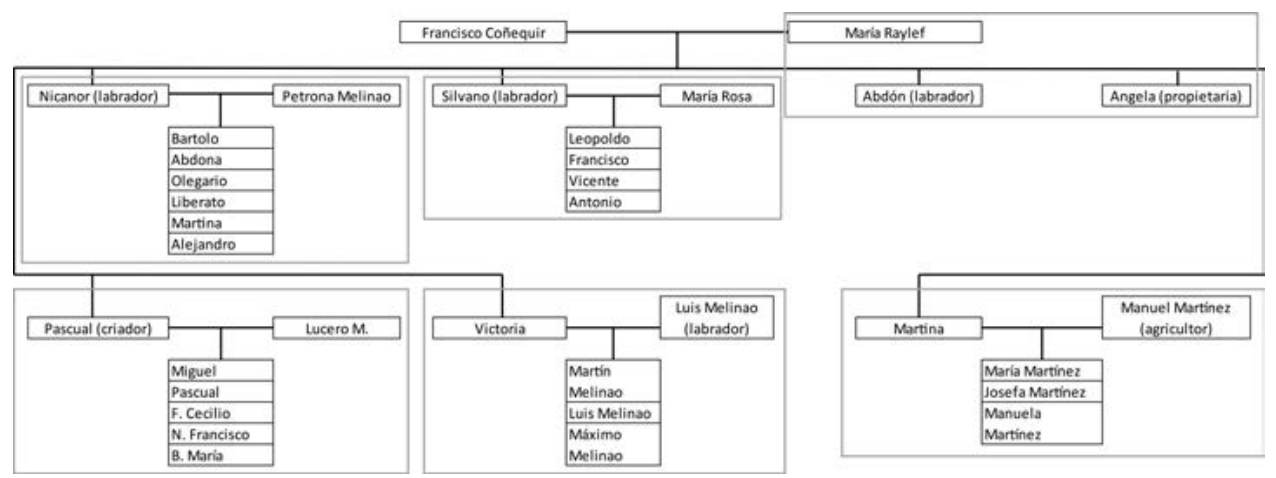

Fuente: elaboración propia en base a ADGPBA, Bragado, $\mathrm{n}^{\circ}$ 98, Pedro Melinao y otros, 1903 $\mathrm{y} \mathrm{n}^{\circ} 100$; Pedro Melinao y otros, 1906. AHMB, Registro de Vecindad, 1890-1891. Censo Nacional de 1895. En línea continua se identifican las unidades domésticas.

Lo que puede comenzar a reconstruirse con mayor certeza es que Cayetano Raylef participó como hombre de lanza del piquete de la tribu desde 1864 hasta 1869 , cuando este fue licenciado y disuelto. Según Hux era hijo del cacique José María Raylef. En 1874 sirvió como alférez en la fuerza que al mando de Pedro Melinao fue agregada a la expedición de Hilario Lagos. Hacia 1890 estaba casado con Justa Olguín y junto a sus hijos José María y Cayetano se dedicaba a la labranza de una unidad doméstica que completaban cuatro niños y la hija mayor Visitación. Esta chacra se ubicaba en un área que se extendía de sur a norte orillando primero las tierras de los Melinao y Coñequir, y después de los Nahuel, Cañiú y Llublicán. Salvo la fracción de Pedro Escalada y la contigua de José Larrea, los descendientes de Raylef se concentraron en esta lonja larga y angosta en la sección más oriental de $\mathrm{La}$ Barrancosa. En ella constó, además, la ocupación de Wenceslao Banega, María Raylef de Morales y Bernarda Pereyra de Raylef (mapa 3, posiciones 13, 14 y 15). En todos estos casos primó una notoria vaguedad de la titularidad y sucesión. El caso de los Raylef muestra además la profusión de unidades mixtas de familias indígenas y población criolla, la inserción laboral jornalera y la difusión del sub-arrendamiento y la medianería; todos elementos que probablemente colaboraron en la enajenación posterior de la tierra.

Banega era un jornalero que junto a otros migrantes de las provincias argentinas de Santiago del Estero y Corrientes fue censado en 1895 junto al labrador español Manuel Martínez y su esposa Martina Coñequir. Es factible que fuera puestero. María Raylef, por su parte, estaba casada con el labrador Morales 
Aguilar y con él vivía junto a sus tres hijos, probablemente un hermano menor del esposo y su propio hermano, Anastasio Raylef, también labrador. Bernarda Pereyra $^{32}$, viuda de un Raylef, fue reconocida como propietaria y hacia 1895 aparentemente vivía con el jornalero Agapito González y sus dos hijas.

Más allá de las familias Melinao, Raylef y Coñequir existió un amplio, diverso y elusivo universo social que pobló La Barrancosa, cuyos contornos étnicos las más de las veces fue difuso. En primer lugar, hay que mencionar que, en estos casos, en términos generales, la fragmentación de la propiedad es mayor. Una treintena de poblaciones y más de medio centenar de lotes circunscribían dos mil quinientas hectáreas según las mensuras de principios de siglo $\mathrm{XX}$. En segundo lugar, que no existe un contraste significativo entre propietarios y posesores. Es decir, las posesiones de fracciones más pequeñas parecen haber tenido mayor éxito en traducir su posesión en derechos de propiedad $-\mathrm{O}$ al menos su reconocimiento catastral - con respecto a fracciones donde es difícil encontrar a sub-arrendatarios, puesteros o medieros. En tercer lugar, que al igual que lo sucedido con las tierras de las grandes familias, la distribución de la posesión - e incluso de la propiedad - esbozó nodos claramente identificables en términos de familias; algo especialmente visible con los Sánchez, Nahuel, Cañiú, Güayquilao y Barrancos, de sur a norte, por ejemplo (ver mapa 3). En cuarto lugar, que estos titulares de tierras emplearon los mismos instrumentos para la cría y comercialización del ganado, tal como veremos a continuación.

CRÍA Y COMERCIALIZACIÓN AGRÍCOLA-GANADERA: LA INSERCIÓN ECONÓMICA

Una parte importante de la información sobre el uso de las tierras de «indios amigos» proviene de las guías de campaña, los boletos de señales de marca para ganado y una diversidad de registros locales creados con el objetivo de instrumentar la recaudación impositiva y resolver litigios en los cuarteles del ámbito rural de los partidos. Ahora bien, es preciso recordar que no todas las actividades productivas y comerciales fueron registradas y que ni siquiera todas las actividades se hicieron en la esfera mercantil ${ }^{33}$.

Las guías de campaña son los registros labrados en el ámbito más capilar de la organización política de las llanuras pampeanas — los juzgados de paz-

32 Pereyra era una familia muy emparentada con los Raylef. Por entonces, por ejemplo, existió un puesto de Ramón Pereyra dedicado a la cría ganadera, quien vivía con su esposa e hijos junto a la familia de Miguel y Agustina Raylef y sus hijos dedicados por entero al trabajo como jornaleros.

33 Literas, 2018b. 
con motivo de identificar las marcas empleadas por los vecinos para el ganado vacuno, caballar y ovino. Los boletos de señales de marca son los documentos emitidos en función de esa identificación, que debía solicitar y emplear el propietario y aceptar y registrar el juzgado de paz para habilitar la cría, el transporte y comercio de animales. A diferencia de la tribu de Rondeau, por ejemplo, donde el uso de estos instrumentos se concentró en los caciques, aquí se observa una importante difusión. En La Barrancosa hubo al menos treinta titulares de una decena de familias propietarias de marcas de ganado. Esta mayor difusión, excediendo ampliamente la gestión y administración de los caciques, sugiere diferentes grados de intervención y mediación de los líderes, un aspecto sobre el que deberá avanzarse en futuros estudios.

En términos generales el análisis de estos instrumentos en La Barrancosa sugiere una intensa actividad pastoril, la combinación de la explotación vacuna, lanar y caballar, y la venta regular a mercados regionales como Buenos Aires. También nos habla de la circulación de ganado entre diferentes propiedades indígenas - por ejemplo, entre las tierras de la tribu de Melinao y la vecina tribu de Coliqueo-, algo a lo que le subyacieron lazos políticos y parentales, y ofrece algunos indicios de cultivos agrícolas con fines comerciales. Sin embargo, como se adelantó en otro lugar, en muchos casos las reducidas extensiones de los lotes — aparejadas a la fragmentación- debieron atentar contra la reproducción de las unidades domésticas ${ }^{34}$.

Las particularidades con respecto a la posesión y la propiedad de $\mathrm{La} \mathrm{Ba}$ rrancosa no significaron que fuera, en términos económicos, una especie de «isla». Al contrario, existió una articulación no menor con la economía local y regional. Con respecto a la primera, los registros documentales indican el suministro en varias ocasiones de ganado vacuno por parte de Pedro Melinao al corral de abasto de Bragado y de modo muy temprano, ya en $1860^{35}$. Esto se observó también para la vecina tribu de Rondeau, en Veinticinco de Mayo. Al parecer el cacique disponía además de una carnicería en La Barrancosa, sobre la cual el municipio de Bragado intentó no siempre con éxito gravar impuestos. A su vez, en el caso de la tribu de Melinao se identificaron otras operaciones al corral de abasto, más allá del cacique. En diferentes ocasiones también lo hicieron Naucufil - con la marca de ganado de Andrés Tatalao, antiguo cabo del piquete de la tribu —, Martín Melinao — con la marca de Pedro Melinao-, Antonio Pascual Tatalao, Dionisio y Mariano Sánchez, Gabriel y Juan Nagüel, y Flora Sánchez. Es decir, el ganado criado en las tierras de la tribu era vendido para el consumo de los vecinos de Bragado. La me-

\footnotetext{
34 Literas, 2016.

35 AHMB, 6, 1, 20, Libro de los corrales, 1860.
} 
moria oral de algunos de los descendientes de los pobladores de La Barrancosa también alude a la venta regular de hortalizas, que eran transportadas por el ferrocarril a Bragado desde la estación Olascoaga.

Con respecto a la integración regional, se dio principalmente en virtud de la ganadería ovina - algo que también apoya la historia oral-, conjuntamente con la explotación yeguariza y agrícola ${ }^{36}$. Los Melinao, Nagüel y Platero vendieron ganado a través de Antonio Yamuzzi a la firma de consignatarios «Ghiraldo y Murature», que tuvo como destino el mercado de Buenos Aires. Solo entre 1874 y 1876 se identificaron operaciones por más de dos mil cueros lanares y mil arrobas de lana ovina, además de un centenar de cueros vacunos y yeguarizos. Yamuzzi registró muchas más operaciones de compra-venta para suministrar a Buenos Aires, especialmente de hacienda ovina, en algunas ocasiones de ganado propio, en otras - como en el caso de la tribu de Melinao- de otros propietarios. Existen indicios de que Yamuzzi tenía una relación con la tribu que excedía estas operaciones ya que arrendaba tierras a los Coñequir en La Barrancosa e incluso intercedió ante la justicia de Bragado en favor de Pedro Melinao. Por su parte, "Ghiraldo y Murature» era una firma relativamente importante de comercializadores de ganado en la provincia, conectada también al mercado internacional, probablemente de Juan A. Ghiraldo y Francisco Murature, residentes en la ciudad de Buenos Aires. A su vez, también se detectaron intercambios asociados a la agricultura. Este fue el caso de Abdón Coñequir, hijo del capitanejo Francisco Coñequir y poblador de una fracción que en las mensuras aparece a título de Andrés Tatalao (mapa 3, posición 9), que vendió trigo a la compañía Dellepiane también con destino a Buenos Aires.

Sin embargo, como se adelantó, no todas las actividades económicas en La Barrancosa son legibles en los registros documentales; algo no exclusivo de este caso. Un modo de avanzar en el conocimiento de estas otras prácticas son los conflictos y litigios ocurridos en el marco de la administración política local, en este caso el juzgado de paz y el municipio de Bragado. Al respecto la información es escasa y fragmentada, y habilita solo aproximaciones indiciarias.

Fueron muy frecuentes los conflictos por la cría y comercialización de ganado en La Barrancosa. Al respecto hemos identificado denuncias de abigeato - robo de ganado - que tuvieron como protagonistas a población indígena $^{37}$ : a Cayetano Raylef en 1880, Pedro Melinao en 1881 y 1884, Gabriel

36 AHMB, 6, 1, 11, Guías de campaña; 6, 1, 12, Guías de campaña; 6, 1, 46, Guías de campaña; 6, 1, 12, Guías de campaña.

37 AHMB, 6, 1, 1, Inventario. 
y Alberto Nagüel en 1885, Liberato Coliqueo, Dionisio Coliqueo y Silverio Melinao en 1899, Pedro Melinao, Silverio Melinao y Eugenio Coliqueo en 1887 y Anastasio Raylef en 1890, entre otros ${ }^{38}$. De todas ellas es preciso detenerse en la primera ya que nos habla del conflicto entre uno de los hijos del difunto cacique José María Raylef y una de las familias terratenientes más poderosas de Buenos Aires, los Unzué ${ }^{39}$, así como del funcionamiento de la administración político-judicial y el papel que jugó el cacique Pedro Melinao.

El mayordomo de Mariano Unzué acusó a Cayetano Raylef de «robarle» hacienda lanar de su establecimiento por valor de diez mil pesos ${ }^{40}$. El juez de paz habría verificado este hurto y apresado a Raylef, por entonces confinado en la cárcel de Bragado, que recibió la condena culpable de abigeato. A sugerencia del mayordomo, el juez de paz embargó doscientas yeguas, una tropilla de caballos y dos bueyes que Raylef conservaba en Nueve de Julio para subsanar las pérdidas ocasionadas a Unzué. Raylef permaneció en la cárcel, aún enfermo, hasta que el cacique Pedro Melinao pagó una fianza de cuatro mil pesos para conseguir su excarcelación.

Durante gran parte del siglo XIX la administración política y militar de los pueblos de la campaña y de las comandancias fronterizas tuvo serias dificultades para controlar las tierras de «indios amigos» y a sus habitantes. Son numerosos los casos en que funcionarios estatales denunciaron que en esas tierras se refugiaban desertores del ejército, se pastoreaba ganado «robado» en los establecimientos criollos o se tramaban alianzas políticas contrarias al Estado. La Barrancosa no fue la excepción y aún para 1887, concluida la Conquista del Desierto, la intendencia de Bragado insistía en la necesidad de «vigilar permanentemente las tolderías de indios de Melinao y Coliqueo, foco perenne de disturbios y raterías» ${ }^{41}$.

\section{FRAGMENTACIÓN Y ENAJENACIÓN: UNA MIRADA EXPLORATORIA}

Como se adelantó no es el propósito aquí reconstruir y analizar la fragmentación y enajenación de las tierras de la tribu de Melinao, sino la ocupa-

38 En otras ocasiones no se especificó el origen del conflicto, pero sí su desenlace, por medio de litigios en virtud de violencia física e incluso el uso de armas. Estos fueron los casos de Antonio Antemil y Silvano Coñequir en 1890, de Cayetano Raylef en 1894, de Liberato Coliqueo y Crispín en 1903, de Juan Cañiú en 1905.

39 Para un análisis de la estructura de la propiedad en el marco del avance fronterizo hacia el sur y el papel de los Unzué, remitimos a Valencia, 2005.

40 AHMB, 6, 1, 25, Libro de actas. 1880.

41 AHMB, Libro de notas del Concejo Deliberante. Año 1886 al 1893, f. 64. 
ción y el uso que de ella hicieron sus legítimos e iniciales adjudicatarios. No obstante, es conveniente avanzar en los primeros indicios que las fuentes ofrecen sobre la enajenación que le siguió a la fragmentación observada en virtud de los vínculos entre posesión y propiedad.

Como en el caso de la tribu de Coliqueo, la introducción de la noción de propiedad y de los mecanismos mercantiles de apropiación de tierras así como la existencia de intereses espurios externos a la tribu repercutió en la fragmentación y enajenación ${ }^{42}$. A esto debe sumársele otro factor: la vaguedad jurídica y sui generis del origen y el tipo de donación que hizo el gobierno provincial de Buenos Aires, algo especialmente claro, por ejemplo, en los prolegómenos para hacer efectiva la Contribución Directa - impuesto general sobre la tierra - En muchas ocasiones el problema residió en determinar si este tipo de tierras cuajaban en la tipología de derechos de propiedad de la estructura normativa estatal.

A inicios del siglo XX, cuarenta años después de obtenida la donación, Pedro Melinao hizo gestiones para la mensura y división de La Barrancosa. Según Hux el cacique fue mal aconsejado - aunque sin especificar por quién ni por qué- y el procedimiento dio lugar a disensos y distanciamientos intra-étnicos ${ }^{43}$. En ese contexto, terratenientes criollos como Ignacio Echechiquía y Ramón M. Pérez, en connivencia con el escribano José Barrera, consiguieron en 1889 que los tribunales de Justicia del Centro de la provincia de Buenos Aires nombrasen como «únicos miembros de la tribu» a Francisco y Pascual Coñequir, Nahuel y Noguera Güayquilao, Dionisio y Mariano Sánchez, Cayetano Raylef, Martín Barrancos, Andrés Tatalao y Marcelino Ávila, excluyendo al cacique Pedro Melinao. Echechiquía, como se verá a continuación, acabó siendo el titular más importante de tierras de la tribu. Melinao, sin embargo, consiguió que en 1903 se levantara la primera mensura y división de La Barrancosa — protestada por Barrera - que fue aprobada por el gobierno en 1905. Resta aún avanzar en la reconstrucción de estos hechos y el análisis de por qué se dieron dichas diferencias al interior de la tribu.

Otro factor que incidió en la enajenación de tierras fue el matrimonio interétnico, algo asociado a la progresiva desarticulación de las unidades socio-políticas que habían vehiculizado las relaciones con la sociedad estatal desde el Negocio Pacífico de Indios. Dicha afirmación no puede generalizarse y requiere de un estudio sistemático de las relaciones de parentesco y la estructura de propiedad y posesión de las tierras en otras tribus. En el caso de Melinao se han identificado uniones conyugales interétnicas que estuvieron asociadas al reco-

\footnotetext{
42 Fischman y Hernández, 1990.

43 Hux, 2007.
} 
nocimiento ulterior de derechos de propiedad a vecinos no indígenas. Ejemplo de ello son las de Severa Alonso y Martín Torres, Celestina Cristóbal y Cipriano Torres, Margarita Guanquel y Santiago Díaz, María Milla y Juan Rogero, Rosaria Coñequir y José Montiel y sin duda las más significativas la de Martina Coñequir y Manuel Martínez, y de María Raylef y Morales Aguilar. Todas mujeres de familias de la tribu casadas con hombres criollos o extranjeros.

Aquí sostenemos la hipótesis de que la posición de la mujer en la red de relaciones parentales de la tribu, así como los recursos económicos del hombre, fueron clave en la situación de la unión conyugal con respecto a la tierra. La disposición de estos capitales sociales y económicos habrían sido decisivos para hacerse de una chacra o puesto en La Barrancosa o para migrar al ámbito urbano de Bragado o asentarse en otras tierras rurales ${ }^{44}$. Simultáneamente, tener garantizado un acceso estable a la tierra muy probablemente generó las condiciones para la enajenación criolla posterior al ser relevados en las mensuras y hasta incluso obtener títulos. Esto no solo apoya la idea de posesión como vehículo de propiedad, sino que muestra una vez más el peso decisivo del parentesco.

El primer caso es el de Severa Alonso y Celestina Cristóbal, casadas con los hermanos Martín y Cipriano Torres. Ambos matrimonios tuvieron cinco hijos, todos de apellido Torres, y aparentemente formaban una misma unidad doméstica en los puestos de Pedro Melinao (mapa 3, fracción C). Martín y Cipriano Torres eran jornaleros, pero en el censo de 1895 el primero fue reconocido como propietario de tierras. En efecto esta población fue relevada en las mensuras de 1903 y 1906, identificada con Severa Alonso de Torres, titular de derecho de una pequeña y angosta fracción de 27 hectáreas en el sector más oriental de las tierras de la tribu (mapa 3, posición 16).

El segundo caso es el de Margarita Guanquel y Santiago Díaz. Aquí también Santiago fue reconocido como propietario, aunque su oficio era de agricultor, por lo que es posible — según el uso que se le daba a estas categorías en los censos y registros- que sus actividades principales no consistieran en trabajar en otras propiedades. A pesar de que no consta ninguna población a nombre de ninguno de los dos en las mensuras de 1903 y 1906 Margarita Guanquel de Díaz fue reconocida como adjudicataria de una fracción de 27 hectáreas entre las tierras de Raylef y Cayunao.

Los otros dos casos, el de Martina Coñequir y Manuel Martínez y de María Raylef y Morales Aguilar, tienen como protagonistas a mujeres de

44 Esto último pudo haber sido el caso de Rosaria Coñequir y María Milla, casadas con dos jornaleros criollos que vivieron fuera de las tierras de la tribu, aunque en el mismo cuartel. 
dos de las grandes familias de la tribu, casadas con hombres probablemente de cierta posición en la humilde estructura social de este punto de la campaña bonaerense. Como se ha visto, Martina era hija del capitanejo Francisco Coñequir, y María Raylef del cacique José María Raylef. En consecuencia, es posible imaginar que tenían una posición relevante en el parentesco de La Barrancosa. Manuel Martínez era español, se dedicaba a la agricultura y tenía junto a Martina Coñequir una población en las proximidades del Saladillo (mapa 3, fracción $\mathrm{H}$, posición 11), a las orillas de los campos de Pedro Melinao y cerca de las posiciones de sus hermanos Abdón y Angela Coñequir (mapa 3, posiciones 9 y 10), aunque también tuvo residencia en el ejido urbano de Bragado. Allí las mensuras de 1903 y 1906 reconocieron a Martina Coñequir de Martínez la propiedad de 47 hectáreas; la misma extensión que al resto de hijos y viuda de Francisco Coñequir. María Raylef estaba casada con el labrador Morales Aguilar con quien vivía junto a sus tres hijos, un hermano menor de su esposo y un hermano propio - Anastasio Raylef, también labrador - en la extensa y discontinua lonja oriental de tierras ocupadas por los Raylef, próxima a las lagunas encadenadas que llegaban hasta La Barrancosa. Al igual que en los casos anteriores María Raylef fue reconocida adjudicataria del derecho de propiedad de una fracción en las mensuras de 1903 y 1906, en este caso de 42 hectáreas.

Esta enajenación progresiva, paulatina y fragmentaria de La Barrancosa se observa en un registro catastral posterior, de 1912, con motivo de crear un camino hasta la estación de ferrocarril Olascoaga ${ }^{45}$. Allí se hizo un relevamiento territorial y un pedido de permiso a los ocupantes que constata este cambio. La primera y más evidente observación es la nueva nominación del campo: se omitió toda alusión a la entidad tribal y étnica - típica en la segunda mitad del siglo XIX - y fue llamado «colonia La Barrancosa», con una definición de «centro agrícola importante». Es llamativa esta nueva denominación ya que «colonia» era un término reservado a las experiencias de colonización agrícola-ganadera fomentadas por el Estado al menos desde fines de la década de 1870 y que tuvo como destinataria, de diferente modo, a población indígena, criolla y extranjera.

La inspección dio cuenta además del creciente uso del alambrado y las tranqueras y, en consecuencia, la clausura de antiguos caminos («una serie de huellas» dijo el informe) por parte de algunos propietarios. En la exigencia que hizo el gobierno para permitir el libre tránsito por esas huellas, se

\footnotetext{
45 AHMB, Exp. 2796-8/912.
} 
Figura 3. MONUMENTO CONMEMORATIVO SITUAdO EN LAS ANTIGUAS Chacras de MelinaO

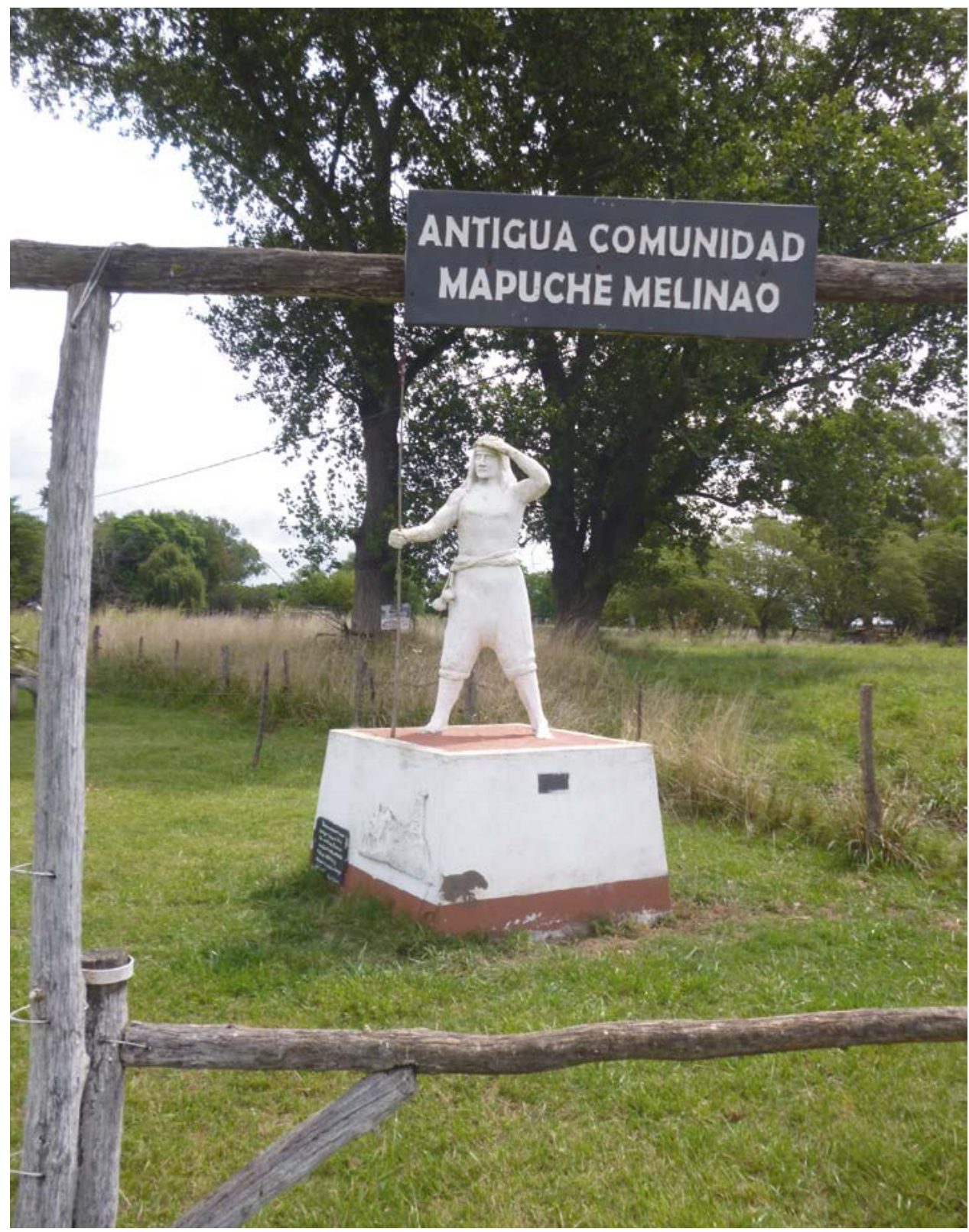

Fuente: fotografía del autor.

Revista de Indias, 2020, vol. LXXX, n. . 280, 781-814, ISSN: 0034-8341

https://doi.org/10.3989/revindias.2020.022 
advierte la presencia de habitantes externos a la tribu que, evidentemente, ya habían adquirido derechos sobre la tierra. Intimó a Pedro Melinao, Antonio Nahuel, Tránsito Cristóbal de Llublicán y Martina Coñequir de Martínez, pero también a los criollos Carlos H. Cigorraga - que vivía en el barrio Montserrat de Buenos Aires-, Carmen Merlo de Molinero, José Larrea y José Montanar.

Además, la inspección produjo un mapa con un relevamiento de la ocupación de La Barrancosa. En él se observa, en primer lugar, que los lotes en la esquina noreste expropiados a fines de extender el ejido de Bragado habían sido, casi en su totalidad, acreditados a un tal Pérez (incluso una fracción aledaña de Florentino Alonso) y otro hombre de apellido de Bollini. Este hecho es al menos llamativo e insinúa irregularidades sobre las que hay que avanzar en el futuro. En segundo lugar, que aparentemente Pedro Melinao adquirió un lote de los Cañiú (el de Manuela) y otro de los Coliqueo (el de Eufemio). También lo hicieron Martina Coñequir sobre los de Sánchez y Collinao, y Ramón Cañiú con el único de los Nehipán. Desconocemos aún la razón de este cambio de titularidad, pero sugiere la transmisión —no sabemos aún cómo- de derechos entre familias indígenas. No obstante, son escasas en comparación con el incremento de operaciones en favor de personas de las que no existe ningún registro de algún tipo sobre relación parental, política o militar siquiera probable con la tribu.

José Larrea, por ejemplo, un pequeño agricultor propietario, para entonces había adquirido las fracciones de Marcos Cayunao y Pedro Escalada y una de los Raylef (ver mapa 4). En este último, caso el origen fue muy probablemente la ocupación y el arrendamiento que había establecido unos años antes con Justa Olguín, viuda de Cayetano Raylef y ahora casada con Nicolás López, con respecto a una fracción «el cual corresponde a la Tribu» ${ }^{46}$. José Montanar había hecho lo propio con la viuda del cacique José María Raylef, Bernarda Pereyra, más al norte y con una fracción de los Cañiú. Por el sudeste, la amplia y angosta lonja de los Raylef había sido cercenada a manos de Luis Arrichaga. En tanto, el criador italiano Félix Abelardo hizo lo propio con las de los Llublicán y de Manquehual Díaz; Ignacio Echechiquía - aquel que había excluido en los tribunales de la provincia a Pedro Melinao- con las de Victoria Coñequir y Luis Melinao; Francisco Sallagai con las de los Tatalao; así como el agricultor francés Bernardo Adoué, Carlos Cigorraga y Augusto Berdia Caula lo hicieron con la fracción más grande de los Sánchez, aledaña a Melinao. El documento arroja indicios, también, sobre la apropiación del

\footnotetext{
46 AHMB, 6, 1, 39, Libro de actas. 1897, f. 233.
} 
rentista Isidoro Molinero sobre las tierras de Barrancos, Sotelo y Güayquilao en torno a la laguna de La Barrancosa.

El catastro posterior de 1920 confirma esta enajenación de La Barrancosa. En este sentido, es preciso mencionar al menos tres aspectos que deben ser profundizados a futuro. En primer lugar, el cambio de propiedad de muchos lotes ya apropiados en años previos por población no indígena, algo que sugiere un aumento de las operaciones mercantiles de lotes. En segundo lugar, el incremento de la apropiación de tierras en pocas manos. En tercer y último lugar, una aceleración de las dinámicas de enajenación y concentración de tierras en no indígenas.

MaPa 4. ENAJENACión de TIERRAS DE LOS RAYLEF

A Título de ArRichaga, Larrea y Montanar, 1912

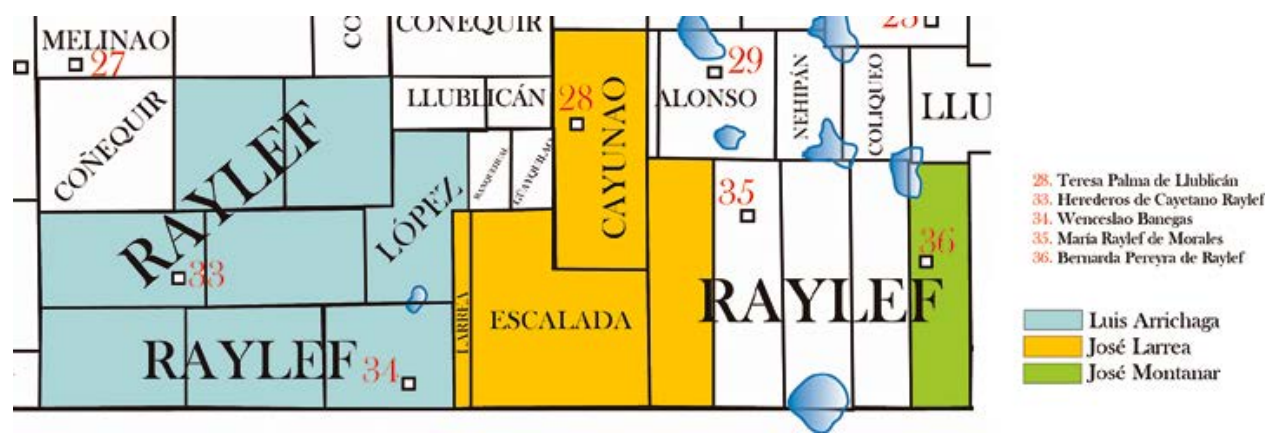

Fuente: elaboración en base Mapa 2 de este manuscrito y de ADGPBA, Bragado, $\mathrm{n}^{\circ}$ 98, $\mathrm{Pe}$ dro Melinao y otros, $1903 \mathrm{y} \mathrm{n}^{\circ} 100$; Pedro Melinao y otros, 1906. AMB, Registro de Vecindad, 1890-1891. AHMB, Exp. 2796-8/912.

\section{CONCLUSIONES}

En La Barrancosa existió una fuerte correlación entre las prestaciones militares en el marco del cuerpo indígena de la tribu y el acceso y uso de $\mathrm{La}$ Barrancosa. Aún a fines del siglo XIX e inicios del siguiente, las mensuras y los registros vecinales evidencian los derechos de propiedad y la ocupación y posesión efectiva de población emparentada y/o descendiente de los miembros de la unidad político-étnica que bajo el liderazgo y las negociaciones del primer cacique Pedro Melinao había obtenido la donación de La Barrancosa en las décadas de 1850 y 1860 . A su vez, existió una lógica de poblamiento con eje en los líderes y según el parentesco que tenían entre sí. Es decir, hubo 
nodos poblacionales en función de la política, el servicio de armas y el parentesco.

Sin embargo, las grandes extensiones de tierra a título de caciques y capitanejos no conllevaron una concentración de su acceso y uso. Las dificultades para conciliar en el análisis la propiedad y la posesión muy probablemente derivaron de que la noción de propiedad fuera al menos extraña a la tribu de Melinao, durante gran parte del siglo XIX; una hipótesis que aún falta comprobar para otras tribus de «indios amigos». No obstante, parece atinada. Esto alude, en definitiva, al conflictivo tránsito de una donación de propiedad colectiva sin mayores precisiones sobre tipo y distribución («al cacique y su tribu»), a la creciente parcelación, mercantilización y privatización del espacio rural insoslayable a la formación y el despliegue de un mercado de tierras.

En La Barrancosa, en efecto, coexistió un heterogéneo mosaico de ocupantes con diferentes lazos y vínculos. Esto refiere, en primer lugar, a la complejidad que adquirieron las relaciones sociales en los campos de la tribu - desde formas de medianería y sub-arrendamiento hasta ocupaciones precarias y trabajo temporal- En segundo lugar, este tipo de poblamiento sugiere la capacidad indígena de incorporar población no indígena mediante el parentesco y los límites heurísticos que plantea la idea de «tribu» para abordar este tipo de problemas de investigación.

Finalmente, fue clara y significativa la inserción económica de las tierras de la tribu en la economía local y regional. La especificidad indígena con respecto a la propiedad, la posesión y el uso de las tierras de la tribu de Melinao y de otros grupos de «indios amigos» no hizo de ellas «islas» desvinculadas de los procesos económicos y sociales más generales, propios de la formación de un modelo de explotación capitalista de la tierra en las llanuras pampeanas. Del mismo modo y en sintonía con lo anterior, La Barrancosa no eludió las dinámicas de mercantilización y transferencia de la tierra, sobre las que es preciso avanzar en próximos estudios.

\section{ARCHIVOS CONSULTADOS}

Archivo de la Dirección de Geodesia de la Provincia de Buenos Aires (ADGPBA).

Archivo Histórico Municipal de Bragado (AHMB).

Archivo General de la Nación (AGN).

Servicio Histórico del Ejército (SHE). 


\section{BIBLIOGRAFÍA}

Barbuto, Lorena, "Iniciativas criollas y territorios indígenas: los proyectos de tierras para los indios amigos de Azul y Tapalqué (1860-1870)", XI Congreso Argentino de Antropología Social, Universidad Nacional de Rosario, 2014.

Bechis, Martha, "Matrimonio y política en la génesis de dos parcialidades mapuche durante el siglo XIX", Martha Bechis, Piezas de etnohistoria y de antropología histórica, Buenos Aires, Sociedad Argentina de Antropología, 2010 [1990], tomo II: 43-64.

Davies Lenoble, Geraldine, Haciéndonos parientes: diplomacia y vida cotidiana entre los linajes indígenas de Nord Patagonia y los criollos de Carmen de Patagones (1852-1879), Tesis de Maestría, Universidad Nacional de Quilmes, 2013.

De Jong, Ingrid, "Funcionarios de dos mundos en un espacio liminal: los «indios amigos» en la frontera de Buenos Aires (1856-1866)", Revista CUSHO, 15 / 2 (Temuco, 2008): 75-95. https://doi.org/10.7770/cuhso-v15n2-art271

De Jong, Ingrid, "El acceso a la tierra entre los indios amigos de la frontera bonaerense (1850-1880)", Revista de Ciencias Sociales (Quilmes, 2015), 27: 87-117.

Fischman, Gustavo y Hernández, Isabel, La ley y la tierra. Historia de un despojo en la tribu mapuche de Los Toldos. Buenos Aires, Buenos Aires, Centro Editor de América Latina, 1990.

Ginzburg, Carlo, El queso y los gusanos. El cosmos, según un molinero del siglo XVI, Barcelona, Muchnik Editores, 2011 [1976].

Ginzburg, Carlo y Poni, Carlo, "El nombre y el cómo. Intercambio desigual y mercado historiográfico", Carlo Ginzburg, Tentativas, Rosario, Prohistoria, 2004 [1979]: 57-67.

Hux, Meinrado, Caciques borogas y araucanos, Buenos Aires, El Elefante Blanco, 2004 [1992].

Hux, Meinrado, Caciques y capitanejos de las llanuras del Plata, Manuscrito, 2007.

Lanteri, Sol y Pedrotta, Victoria "Tierras, armas y política en la frontera sur bonaerense durante la década de 1850. Los «indios amigos», Maicá, Villa Fidelidad", Anuario del Instituto de Historia Argentina, 18/1 (Buenos Aires, 2018): e066. https://doi.org/10.24215/2314257xe066

Lanteri, Sol, Ratto, Silvia, De Jong, Ingrid y Pedrotta, Victoria, “Territorialidad indígena y políticas oficiales de colonización. Los casos de Azul y Tapalqué en la frontera sur bonaerense (siglo XIX)", Antiteses, 4 /8 (Londrina, 2011): 729-752. https://doi.org/10.5433/1984-3356.2011v4n8p729 
Literas, Luciano, "De donaciones, arrendamientos y compras. Acceso y uso de la tierra de los indios amigos (la tribu de Rondeau, segunda mitad siglo XI)", Publicar, XIII/18 (Buenos Aires, 2015): 59-84.

Literas, Luciano, "De litigios, recursos y sumarios. La propiedad de la tierra en la tribu de Melinao (Buenos Aires, segunda mitad siglo XIX)", Memoria Americana, 24/2 (Buenos Aires, 2016): 59-84.

Literas, Luciano, "De parientes, conflictos y memorias. Las iniciativas de acceso a la tierra del cacique Andrés Raninqueo (Buenos Aires, segunda mitad del siglo XIX)", Tefros, 16/1 (Río Cuarto, 2018a): 9-33.

Literas, Luciano, "Metodología y fuentes para el estudio del acceso y el uso indígena de la tierra. Las llanuras pampeanas, siglo XIX", $56^{\circ}$ Congreso Internacional de Americanistas, Salamanca, Universidad de Salamanca, 2018b.

Literas, Luciano y Barbuto, Lorena, "El acceso a la tierra de los indios amigos. Una comparación preliminar de las tribus de Catriel y Rondeau (Buenos Aires, segunda mitad del siglo XIX)", Tefros, 13/2 (Río Cuarto, 2015): 149-170.

Literas, Luciano y Barbuto, Lorena, "Más allá de los caciques. Los hombres de lanza en las fuentes seriadas de la militarización indígena (Pampa y Nor-Patagonia, siglo XIX)", III Congreso Internacional: Nuevos Horizontes de Iberoamérica, Cuyo, Universidad Nacional de Cuyo, 2017.

Martinelli, María Laura, “Construcción estatal e «indios amigos»: el acceso a la tierra de la tribu de Ancalao en el enclave fronterizo de Bahía Blanca”, Memoria Americana, 25/1 (Buenos Aires, 2017): 97-114.

Martinelli, María Laura, De «tierra adentro» a la frontera: cambios y continuidades en la conformación de «tribus de indios amigos» en Bahía Blanca (1830-1880), Buenos Aires, Universidad de Buenos Aires, 2018.

Quijada, Mónica, "La lenta configuración de una "Ciudadanía cívica» de frontera. Los indios amigos de Buenos Aires, 1820-1879 (con un estudio comparativo Estados Unidos-Argentina)", Mónica Quijada (ed.), De los cacicazgos a la ciudadanía. Sistemas políticos en la frontera, Río de la Plata, siglos XVIII-XX, Berlín, Ibero-Amerikanisches Institut, 2011: 149-308.

Ratto, Silvia, "Una experiencia fronteriza exitosa: el Negocio Pacífico de Indios en la provincia de Buenos Aires (1829-1852)", Revista de Indias, LXIII/227 (Madrid, 2003): 191-222. https://doi.org/10.3989/revindias.2003.i227.437

Salomón Tarquini, Claudia, Largas noches en La Pampa. Itinerarios y resistencias de la población indígena, 1878-1976, Buenos Aires, Prometeo, 2010.

Salomón Tarquini, Claudia, Rollhauser, Elisabet y Nagy, Mariano, "Trabajo y trayectorias familiares de indígenas en Pampa y oeste de Buenos Aires (Argentina, 1882-1920)", Revista Mundos do Trabalho, 6/12 (Santa Catarina, 2014): 153-173. https://doi.org/10.5007/1984-9222.2014v6n12p153 
Valencia, Marta, Tierras públicas, tierras privadas. Buenos Aires, 1852-1876, La Plata, Universidad Nacional de La Plata, 2005.

Villar, Daniel y Jiménez, Juan Francisco, “Amigos, hermanos y parientes. Líderes y liderados en la Pampa Centro Oriental (1820-1840). Etnogénesis Llailmache”, Daniel Villar y Juan Francisco Jiménez (eds.) Amigos, hermanos y parientes. Lideres y liderados en las Sociedades Indígenas de la pampa oriental (siglo XIX), Bahía Blanca, Centro de Documentación Patagónica. Departamento de Humanidades, Universidad Nacional del Sur, 2011: 115-170.

Fecha de recepción: 2 de noviembre de 2018.

Fecha de envío de las modificaciones: 26 de marzo de 2019.

Fecha de aceptación: 10 de abril de 2019.

\section{State, market and indigenous land use: La Barrancosa (Buenos Aires, 1863-1906)}

The following article analyses the indigenous possession and use of land in the province of Buenos Aires (Argentina), within the framework of state organization and the formation of the land market in the second half of the nineteenth century. To do so, it deals with the case of the Melinao tribe and the lands on which they settled in La Barrancosa (Bragado, Buenos Aires), the property they received as a donation in 1863. The analysis is based on the triangulation of a heterogeneous set of documentary sources about population, service of arms, kinship, lands, production and commerce.

Key words: Pampas and North Patagonia; indigenous people; friendly Indians; land market; state organization. 